Research Article

\title{
Square-Mean Pseudo Almost Periodic Solutions for Quaternion-Valued Stochastic Neural Networks with Time-Varying Delays
}

\author{
Yuanyuan Hou (iD) ${ }^{1}$ and Lihua Dai $\mathbb{D}^{1,2}$ \\ ${ }^{1}$ Department of Mathematics and Statistics, Puer University, Puer, Yunnan 665000, China \\ ${ }^{2}$ School of Mathematics and Statistics, Southwest University, Chongqing 400715, China \\ Correspondence should be addressed to Lihua Dai; hlm2136816@163.com
}

Received 29 October 2020; Revised 3 January 2021; Accepted 6 January 2021; Published 21 January 2021

Academic Editor: Francisco Rodríguez

Copyright (C) 2021 Yuanyuan Hou and Lihua Dai. This is an open access article distributed under the Creative Commons Attribution License, which permits unrestricted use, distribution, and reproduction in any medium, provided the original work is properly cited.

\begin{abstract}
In this paper, we are concerned with a class of quaternion-valued stochastic neural networks with time-varying delays. Firstly, we cannot explicitly decompose the quaternion-valued stochastic systems into equivalent real-valued stochastic systems; by using the Banach fixed point theorem and stochastic analysis techniques, we obtain some sufficient conditions for the existence of squaremean pseudo almost periodic solutions for this class of neural networks. Then, by constructing an appropriate Lyapunov functional and stochastic analysis techniques, we can also obtain sufficient conditions for square-mean exponential stability of the considered neural networks. All of these results are new. Finally, two examples are given to illustrate the effectiveness and feasibility of our main results.
\end{abstract}

\section{Introduction}

It is well known that the dynamic research on neural network models has achieved fruitful results, and it has been widely used in the fields of pattern recognition, automatic control, signal processing, and artificial intelligence. However, most neural network models proposed and discussed in the literature are deterministic. It has the characteristics of simple and easy to analyze. In fact, for any actual system, there is always a variety of random factors. As we know, in real nervous systems and in the implementation of artificial neural networks, noise is unavoidable $[1,2]$ and should be taken into consideration in modelling. Stochastic neural network is an artificial neural network and is used as a tool of artificial intelligence. Therefore, it is of practical importance to study the stochastic neural networks. In 1996, Liao and Mao [3] studied stochastic effects to the stability property of a neural network. Subsequently, some scholars carried out a lot of research work and made some progress [4-7]. Due to the finite switching speed of neurons and amplifiers, time delays inevitably exist in biological and artificial neural network models. In recent years, the research on the stability of delay stochastic neural networks has become a hot spot in many scholars [8-11].

On the one hand, the quaternion-valued neural network has been one of the most popular research hot spots, due to the storage capacity advantage compared to real-valued neural networks and complex-valued neural networks. It can be applied to the fields of robotics, attitude control of satellites, computer graphics, ensemble control, color night vision, and image impression [12, 13]. Because all of these applications rely heavily on their dynamics, the study of various dynamical behaviors for quaternion-valued neural networks has received much attention of many scholars, and some results have been obtained for the stability [14-16], dissipativity [17], and pseudo almost periodicity $[18,19]$ of quaternion-valued neural networks. In recent years, authors of $[20,21]$ considered the existence and global exponential stability of pseudo almost periodic solutions and pseudo almost automorphic solutions for quaternion-valued neural 
networks by direct method. It should be noted that most studies on quaternion-valued neural network dynamic behaviors are concerned with the quaternion-valued deterministic neural networks, and so far, only a few results consider the stochastic quaternion-valued neural networks via a decomposing method [22, 23]. For example, Sriraman et al. [22] considered the square-mean asymptotic stability for the discrete-time stochastic quaternion-valued neural networks; in [23], authors studied the square-mean exponential input-to-state stability for the continuous-time stochastic memristive quaternion-valued neural networks.

On the other hand, pseudo almost periodicity is a natural generalization of almost periodicity. Therefore, for nonautonomous neural networks, pseudo almost periodic oscillation is a very important dynamic phenomenon. In the past few decades, many researchers have investigated various dynamical behaviors of stochastic differential equations such as the existence and stability of almost periodic solutions [24], almost automorphic solutions [25, 26], and pseudo almost periodic solutions $[27,28]$. Besides, as we all know, there have been only few results about the dynamic behaviors of nonautonomous stochastic neural networks. Subsequently, some scholars have studied the existence and stability of periodic solutions and almost periodic solutions for stochastic neural networks [29-31], for example, Lu and $\mathrm{Ma}$ [29] dealt with the stability analysis and the existence of periodic solution problems for the stochastic neural networks; Huang and Yang [31] investigated the problems of existence of quadratic mean almost periodic and global exponential stability for stochastic cellular neural networks with delays. Compared with the previous results, rare results are available for pseudo almost periodic solutions of stochastic neural networks in the mean square sense.

However, to the best of our knowledge, up to now, there is no paper published on the existence and square-mean exponential stability for square-mean pseudo almost periodic solutions of quaternion-valued stochastic neural networks. So, it is a challenging and important problem in theories and applications.

Motivated by the above, in this paper, we consider the following quaternion-valued stochastic neural network:

$$
\begin{aligned}
\mathrm{d} u_{l}(t)= & {\left[-\alpha_{l}(t) u_{l}(t)+\sum_{k=1}^{n} b_{l k}(t) f_{k}\left(u_{k}(t)\right)+\sum_{k=1}^{n} c_{l k}(t) g_{k}\left(u_{k}\left(t-v_{l k}(t)\right)+\mathscr{I}_{l}(t)\right] \mathrm{d} t\right.} \\
& +\sum_{k=1}^{n} \delta_{l k}\left(u_{k}\left(t-\eta_{l k}(t)\right)\right) \mathrm{d} W_{k}(t),
\end{aligned}
$$

where $l \in\{1,2, \ldots, n\}=: \mathscr{T}$; $n$ is the number of neurons in layers; $u_{l}(t) \in \mathbb{Q}$ is the state of the $l$-th neuron at time $t$; $\alpha_{l}(t)>0$ is the self-feedback connection weight; $b_{l k}(t)$ and $c_{l k}(t) \in \mathbb{Q}$ are, respectively, the connection weight and the delay connection weight from neuron $k$ to neuron $l ; v_{l k}(t)$ and $\eta_{l k}(t)$ are the transmission delays; $f_{k}, g_{k}: \mathbb{Q} \longrightarrow \mathbb{Q}$ are the activation functions; $\mathscr{I}_{l}(t) \in \mathbb{Q}$ is an external input on the $l$-th unit at time $t ; W(t)=\left(W_{1}(t), W_{2}(t), \ldots, W_{n}(t)\right)^{T}$ is an $n$-dimensional Brownian motion defined on a complete probability space; $\delta_{l k}: \mathbb{Q} \longrightarrow \mathbb{Q}$ is a Borel measurable function; $A=\left(\delta_{l k}\right)_{n \times n}$ is the diffusion coefficient matrix.

The skew field of quaternion by

$$
\mathbb{Q}:=\left\{q=q^{R}+i q^{I}+j q^{J}+k q^{K}\right\}
$$

where $q^{R}, q^{I}, q^{J}$, and $q^{K}$ are real numbers; the three imaginary units $i, j$, and $k$ obey the Hamilton's multiplication rules:

$$
\begin{aligned}
& i j=-j i=k, \\
& j k=-k j=i, \\
& k i=-i k=j, \\
& i^{2}=j^{2}=k^{2}=i j k=-1 .
\end{aligned}
$$

For every $u \in \mathbb{Q}$, the conjugate transpose of $u$ is defined as $u^{*}=u^{R}-i u^{I}-j u^{J}-k u^{K}$, and the norm of $u$ is defined as

$$
\|u\|_{\mathbb{Q}}=\sqrt{u u^{*}}=\sqrt{\left(u^{R}\right)^{2}+\left(u^{I}\right)^{2}+\left(u^{J}\right)^{2}+\left(u^{K}\right)^{2}} .
$$

For every $u=\left(u_{1}, u_{2}, \ldots, u_{n}\right)^{T} \in \mathbb{Q}^{n}$, we define $\|u\|_{\mathbb{Q}^{n}}=\max _{l \in \mathscr{T}}\left\{\left\|u_{l}\right\|_{\mathbb{Q}}\right\}$.

Let $\left(W, \mathscr{F},\left\{\mathscr{F}_{t}\right\}_{t \geq 0}, P\right)$ be a complete probability space with a natural filtration $\left\{\mathscr{F}_{t}\right\}_{t \geq 0}$, satisfying the usual conditions (i.e., it is right continuous, and $\mathscr{F}_{0}$ contains all P-null sets). Denote by $\mathrm{BC}_{\mathscr{F}_{0}}\left([-v, 0], \mathbb{Q}^{n}\right)$ the family of all bounded, $\mathscr{F}_{0}$-measurable, $C\left([-v, 0], \mathbb{Q}^{n}\right)$-valued random variables $\phi$. Denote by $\mathscr{L}_{\mathscr{F}}^{2}\left([-v, 0], \mathbb{Q}^{n}\right)$ the family of all $\mathscr{F}_{0}$-measurable, $C\left([-v, 0], \mathbb{Q}^{n}\right)$-valued random variables $\phi$, satisfying $\sup _{s \in[-\gamma, 0]} E|\phi(s)|^{2}<\infty$, where $E$ denotes the expectation of stochastic process.

For the convenience, we will adopt the following notation:

$$
\begin{aligned}
\alpha_{l}^{-} & =\inf _{t \in \mathbb{R}} \alpha_{l}(t), \\
b_{l k}^{+} & =\sup _{t \in \mathbb{R}}\left\|b_{l k}(t)\right\|_{\mathbb{Q}^{\prime}} \\
c_{l k}^{+} & =\sup _{t \in \mathbb{R}}\left\|c_{l k}(t)\right\|_{\mathbb{Q}^{\prime}} \\
v^{+} & =\max _{l . k \in \mathscr{T}}\left\{\sup _{t \in \mathbb{R}} v_{l k}(t)\right\}, \\
\eta^{+} & =\max _{l, k \in \mathscr{T}}\left\{\sup _{t \in \mathbb{R}} \eta_{l k}(t)\right\}, \\
\nu & =\max \left\{v^{+}, \eta^{+}\right\} .
\end{aligned}
$$


The initial conditions of system (1) are of the form

$$
u_{l}(s)=\phi_{l}(s), \quad s \in[-v, 0],
$$

where $\phi_{l} \in \mathrm{BC}_{\mathscr{F}_{0}}([-v, 0], \mathbb{Q}), l \in \mathscr{T}$.

Remark 1. Quaternion-valued stochastic system (1) includes real-valued stochastic systems and complex-valued stochastic systems as its special cases. In fact, in system (1),

(i) If all the coefficients and delays are functions from $\mathbb{R}$ to $\mathbb{R}$, and all the activation functions are functions from $\mathbb{R}$ to $\mathbb{R}$, then the state $u_{l}(t) \equiv u_{l}^{R}(t) \in \mathbb{R}$; in this case, system (1) is a real-valued stochastic system;

(ii) If the coefficients are functions from $\mathbb{R}$ to $\mathbb{C}$, and all the activation functions are functions from $\mathbb{C}$ to $\mathbb{C}$, then the state $u_{l}(t) \equiv u_{l}^{R}(t)+i u_{l}^{I}(t) \in \mathbb{C}$; in this case, system (1) is a complex-valued stochastic system.

With the inspiration from the previous research, in order to fill the gap in the research field of quaternion-valued stochastic neural networks, the work of this article comes from two main motivations. (1) In practical applications, pseudo almost periodic motion is an interesting and significant dynamical property for stochastic differential equations. In the past decade, many authors studied squaremean almost periodic oscillations and square-mean almost automorphic oscillations of stochastic differential equations [24-26]. Yet, few literatures considered square-mean pseudo almost periodic oscillation of stochastic differential equations [27, 28]. (2) Recently, a few literatures [22, 23] had studied the square-mean stability of quaternion-valued stochastic neural networks via a decomposing method. It is noteworthy that the scholars have not begun to consider the square-mean pseudo almost periodic oscillation for quaternion-valued stochastic neural networks; thus, it is worth studying square-mean pseudo almost periodic motion of quaternion-valued stochastic neural network models by direct method.

Compared with the previous literatures, the distinct characteristics and main contributions of this article are narrated as follows:

(1) Firstly, to the best of our knowledge, this is the first time to investigate the existence and square-mean exponential stability of square-mean pseudo almost periodic solutions for quaternion-valued stochastic delayed neural networks.
(2) Secondly, the method that we use to quaternionvalued stochastic neural networks is different from that used in $[22,23]$, and we improve the norm.

(3) Thirdly, the techniques of this paper can be applied to study the square-mean pseudo almost periodic solutions for other types of quaternion-valued stochastic neural networks.

(4) Finally, examples and numerical simulations are given to verify the effectiveness of the conclusion.

This paper is organized as follows: In Section 2, we introduce some definitions and some preliminary results. In Section 3, we establish some sufficient conditions for the existence of square-mean pseudo almost periodic solutions of system (1). In Section 4, we obtain the square-mean exponential stability of system (1). In Section 5, we give two examples to demonstrate the feasibility of our results. Finally, we draw a conclusion in Section 6.

\section{Preliminaries and Basic Knowledge}

Assume that $\mathbb{H}$ is a real separable Hilbert space, and $L^{2}(P, \mathbb{W})$ stands for the space of all $\llbracket$-valued random variables $X$ such that $E\left(\|X\|^{2}\right)=\int_{\Omega}\|X\|^{2} \mathrm{~d} P<\infty$. Then, $L^{2}(P, \mathbb{M})$ is a Banach space with the norm $\|X\|_{2}=\left(\int_{\Omega}\|X\|^{2} \mathrm{~d} P\right)^{(1 / 2)}$.

Similar to the definition in [27], we give the following definitions.

Definition 1. A stochastic process $X: \mathbb{R} \longrightarrow L^{2}\left(P, \mathbb{Q}^{n}\right)$ is said to be $L^{2}$-continuous if

$$
\lim _{t \longrightarrow s} \mathbb{E}\|X(t)-X(s)\|_{\mathbb{Q}^{n}}^{2}=0 .
$$

It is $L^{2}$-bounded if $\sup _{t \in \mathbb{R}} \mathbb{E}\|X(t)\|_{\mathbb{Q}^{n}}^{2}<\infty$.

Definition 2. An $L^{2}$-continuous stochastic process $X: \mathbb{R} \longrightarrow L^{2}\left(P, \mathbb{Q}^{n}\right)$ is said to be square-mean almost periodic, if for any $\varepsilon>0$, there exists $L=L(\varepsilon)>0$, such that for any $a \in \mathbb{R}$, there exists $\tau \in[a, a+L]$ with

$$
\sup _{t \in \mathbb{R}} E\|X(t+\tau)-X(t)\|_{\mathbb{Q}^{n}}^{2} \leq \varepsilon .
$$

We denote by $\operatorname{SAP}\left(\mathbb{R}, L^{2}\left(P, \mathbb{Q}^{n}\right)\right)$ the set of all squaremean almost periodic functions from $\mathbb{R}$ to $L^{2}\left(P, \mathbb{Q}^{n}\right)$, and let $\mathrm{BC}\left(\mathbb{R}, L^{2}\left(P, \mathbb{Q}^{n}\right)\right)$ be the set of all bounded continuous stochastic processes from $\mathbb{R}$ to $L^{2}\left(P, \mathbb{Q}^{n}\right)$.

Let

$$
\operatorname{SPAP}_{0}\left(\mathbb{R}, L^{2}\left(P, \mathbb{Q}^{n}\right)\right)=\left\{\left.X \in \mathrm{BC}\left(\mathbb{R}, L^{2}\left(P, \mathbb{Q}^{n}\right)\right)\right|_{T \longrightarrow+\infty} \lim _{2 T} \frac{1}{2 T} \int_{-T}^{T} E\|X(t)\|_{\mathbb{Q}^{n}}^{2} \mathrm{~d} t=0\right\} .
$$

We give the following definition for quaternion. 
Definition 3. An $L^{2}$-continuous stochastic process $X: \mathbb{R} \longrightarrow L^{2}\left(P, \mathbb{Q}^{n}\right)$ is said to be square-mean pseudo almost periodic, if it can be decomposed as $X=Y+Z$, where

$$
\begin{aligned}
& Y \in \operatorname{SAP}\left(\mathbb{R}, L^{2}\left(P, \mathbb{Q}^{n}\right)\right), \\
& Z \in \operatorname{SPAP}_{0}\left(\mathbb{R}, L^{2}\left(P, \mathbb{Q}^{n}\right)\right) .
\end{aligned}
$$

We denote by $\operatorname{SPAP}\left(\mathbb{R}, L^{2}\left(P, \mathbb{Q}^{n}\right)\right)$ the set of all squaremean pseudo almost periodic functions from $\mathbb{R}$ to $L^{2}\left(P, \mathbb{Q}^{n}\right)$.

Similar to the lemma in [27], one can easily show as follows.

Lemma 1. If $f, g \in \operatorname{SPAP}\left(\mathbb{R}, L^{2}(P, \mathbb{Q})\right)$, then $f+g, f g \in$ $\operatorname{SPAP}\left(\mathbb{R}, L^{2}(P, \mathbb{Q})\right)$.

Lemma 2. If $\phi \in \operatorname{SPAP}\left(\mathbb{R}, L^{2}(P, \mathbb{Q})\right), \quad \theta \in S A P\left(\mathbb{R}, L^{2}\right.$ $(P, \mathbb{R})) \cap C^{1}(\mathbb{R}, \mathbb{R})$ with $\vartheta:=\inf _{t \in \mathbb{R}}\left(1-\theta^{\prime}(t)\right)>0$, then $\phi(\cdot-\theta(\cdot)) \in \operatorname{SPAP}\left(\mathbb{R}, L^{2}(P, \mathbb{Q})\right)$.

Throughout the rest of the paper, we assume that

$\left(\mathrm{H}_{1}\right) \alpha_{l} \in \operatorname{SAP}\left(\mathbb{R}, \mathbb{R}^{+}\right), \quad v_{k l}, \eta_{l k} \in \operatorname{SAP}\left(\mathbb{R}, \mathbb{R}^{+}\right) \cap C^{1}$ $(\mathbb{R}, \mathbb{R})$ with $\gamma=\sup _{t \in \mathbb{R}}\left\{v_{k l}^{\prime}(t)\right\}, \beta=\sup _{t \in \mathbb{R}}\left\{\eta_{l k}^{\prime}(t)\right\}$, and $b_{l k}, c_{l k}, \mathscr{I}_{l} \in \operatorname{SPAP}(\mathbb{R}, \mathbb{Q})$, where $l, k \in \mathscr{T}$.

(H2) There exist positive constants $L_{k}^{f}, L_{k}^{g}$, and $L_{l k}^{\delta}$ such that for any $x, y \in \mathbb{Q}$,

$$
\begin{gathered}
\left\|f_{k}(x)-f_{k}(y)\right\|_{\mathbb{Q}} \leq L_{k}^{f}\|x-y\|_{\mathbb{Q}}, \\
\left\|g_{k}(x)-g_{k}(y)\right\|_{\mathbb{Q}} \leq L_{k}^{g}\|x-y\|_{\mathbb{Q}}, \\
\left\|\delta_{l k}(x)-\delta_{l k}(y)\right\|_{\mathbb{Q}} \leq L_{l k}^{\delta}\|x-y\|_{\mathbb{Q}},
\end{gathered}
$$

and $f_{k}(0)=g_{k}(0)=\delta_{l k}(0)=0$, where $l, k \in \mathscr{T}$. $\left(\mathrm{H}_{3}\right) \max _{l \in \mathscr{T}}\left\{3 \Upsilon_{l} /\left(\alpha_{l}^{-}\right)^{2}\right\}=: \rho<1$, where

$$
\begin{aligned}
Y_{l}= & \sum_{k=1}^{n}\left(b_{l k}^{+}\right)^{2} \sum_{k=1}^{n}\left(L_{k}^{f}\right)^{2}+\sum_{k=1}^{n}\left(c_{l k}^{+}\right)^{2} \sum_{k=1}^{n}\left(L_{k}^{g}\right)^{2} \\
& +\frac{\alpha_{l}^{-}}{2} \sum_{k=1}^{n}\left(L_{l k}^{\delta}\right)^{2} .
\end{aligned}
$$

(H4) For $l \in \mathscr{T}$, there exist positive constants $\lambda$ and $\xi_{k}$ such that

$$
\begin{aligned}
2 \xi_{l} \alpha_{l}^{-}> & \lambda \xi_{l}+2 \xi_{l}+\sum_{k=1}^{n} \xi_{k}\left(b_{k l}^{+}\right)^{2}\left(L_{k}^{f}\right)^{2}+\sum_{k=1}^{n} \xi_{k}\left(c_{k l}^{+}\right)^{2}\left(L_{k}^{g}\right)^{2} \\
& \frac{e^{\lambda v^{+}}}{1-\gamma}+\sum_{k=1}^{n} \xi_{k}\left(L_{k l}^{\delta}\right)^{2} \frac{e^{\lambda \eta^{+}}}{1-\beta} .
\end{aligned}
$$

\section{Square-Mean Pseudo Almost Periodic Solutions}

In this section, we will study the existence of square-mean pseudo almost periodic solutions of system (1).

Let $\mathscr{X}=\left\{\phi \mid \phi \in \operatorname{SPAP}\left(\mathbb{R}, L^{2}\left(P, \mathbb{Q}^{n}\right)\right)\right.$ with the norm $\|\phi\|_{\mathscr{X}}=\sup _{t \in \mathbb{R}}\left(E\|\phi(t)\|_{\mathbb{Q}^{n}}^{2}\right)^{(1 / 2)}$, and then $\mathscr{X}$ is a Banach space.

$\int^{t} e^{-} \int_{s}^{t} \alpha_{l} \phi^{0}(\theta) \mathrm{d} \theta=\left(\phi_{1}^{0}, \phi_{2}^{0}, \ldots, \phi_{n}^{0}\right)^{T}, \quad$ where $\phi_{l}^{0}(t)=$ stant satisfying $\kappa \geq\left\|\phi^{0}\right\|_{\mathscr{X}}$.

Theorem 1. Let $\left(H_{1}\right)-\left(H_{3}\right)$ hold. Then, system (1) has a unique square-mean pseudo almost periodic solution in the region $\mathscr{X}^{*}=\left\{\phi|\phi \in \mathscr{X}|\left\|\phi-\phi^{0}\right\|_{\mathscr{X}} \leq \kappa\right\}$.

Proof. Firstly, it is easy to see that if $u(t)=\left\{\left(u_{1}(t)\right.\right.$, $\left.\left.u_{2}(t), \ldots, u_{n}(t)\right)^{T}\right\}_{t \in \mathbb{R}}$ is a solution of the following stochastic integral equation

$$
\begin{aligned}
u_{l}(t)= & \int_{-\infty}^{t} e^{-\int_{s}^{t} \alpha_{l}(\theta) \mathrm{d} \theta}\left[\sum_{k=1}^{n} b_{l k}(s) f_{k}\left(u_{k}(s)\right)+\sum_{k=1}^{n} c_{l k}(s) g_{k}\left(u_{k}\left(s-v_{k l}(s)\right)\right)+\mathscr{I}_{l}(s)\right] \mathrm{d} s \\
& +\int_{-\infty}^{t} e^{-\int_{s}^{t} \alpha_{l}(\theta) \mathrm{d} \theta} \sum_{k=1}^{n} \delta_{l k}\left(u_{k}\left(s-\eta_{l k}(s)\right)\right) \mathrm{d} W_{k}(s),
\end{aligned}
$$

where $l \in \mathscr{T}$, then $u$ is also a solution of system (1).

We define a mapping $\Phi: \mathscr{X} \longrightarrow \mathscr{X}$ by setting

$$
\left(\phi_{1}, \phi_{2}, \ldots, \phi_{n}\right) \longrightarrow\left(u_{1}^{\phi}, u_{2}^{\phi}, \ldots, u_{n}^{\phi}\right) .
$$

We shall show that $\Phi$ has a unique fixed point in $\mathscr{X}^{*}$. For $\phi \in \mathscr{X}^{*}$, we have

$$
\|\phi\|_{\mathscr{X}} \leq\left\|\phi^{0}\right\|_{\mathscr{X}}+\left\|\phi-\phi^{0}\right\|_{\mathscr{X}} \leq 2 \kappa .
$$

For any $\phi \in \mathscr{X}^{*}$, from (14), we consider the following stochastic integral equation:

$$
\begin{aligned}
u_{l}^{\phi}(t)=: & \int_{-\infty}^{t} e^{-\int_{s}^{t} \alpha_{l}(\theta) \mathrm{d} \theta} F_{l}(s) \mathrm{d} s+\int_{-\infty}^{t} e^{-\int_{s}^{t} \alpha_{l}(\theta) \mathrm{d} \theta} \\
& \cdot \sum_{k=1}^{n} G_{l k}(s) \mathrm{d} W_{k}(s), \quad l \in \mathscr{T},
\end{aligned}
$$


where

$$
\begin{aligned}
F_{l}(s)= & \sum_{k=1}^{n} b_{l k}(s) f_{k}\left(\phi_{k}(s)\right)+\sum_{k=1}^{n} c_{l k}(s) g_{k}\left(\phi_{k}\left(s-v_{k l}(s)\right)\right) \\
& +\mathscr{I}_{l}(s) \\
G_{l k}(s)= & \delta_{l k}\left(\phi_{k}\left(s-\eta_{l k}(s)\right)\right) .
\end{aligned}
$$

From Lemmas 1 and 2, we have $F_{l}, G_{l k} \in \operatorname{SPAP}\left(\mathbb{R}, L^{2}\right.$ $(P, \mathbb{Q})), \quad l, k \in \mathscr{T}$, that is, $F_{l}, G_{l k}$ can be rewritten as $F_{l}=F_{l}^{1}+F_{l}^{0}, \quad G_{l k}=G_{l k}^{1}+G_{l k}^{0}$, where $F_{l}^{1}, G_{l k}^{1} \in \operatorname{SAP}\left(\mathbb{R}, L^{2}\right.$ $(P, \mathbb{Q})), F_{l}^{0}, G_{l k}^{0} \in \operatorname{SPAP}_{0}\left(\mathbb{R}, L^{2}(P, \mathbb{Q})\right)$. Hence,

$$
\begin{aligned}
u_{l}^{\phi}(t)= & \int_{-\infty}^{t} e^{-\int_{s}^{t} \alpha_{l}(\theta) \mathrm{d} \theta} F_{l}^{1}(s) \mathrm{d} s+\int_{-\infty}^{t} e^{-\int_{s}^{t} \alpha_{l}(\theta) \mathrm{d} \theta} \sum_{k=1}^{n} G_{l k}^{1}(s) \mathrm{d} W_{k}(s)+\int_{-\infty}^{t} e^{-\int_{s}^{t} \alpha_{l}(\theta) \mathrm{d} \theta} F_{l}^{0}(s) \mathrm{d} s \\
& +\int_{-\infty}^{t} e^{-\int_{s}^{t} \alpha_{l}(\theta) \mathrm{d} \theta} \sum_{k=1}^{n} G_{l k}^{0}(s) \mathrm{d} W_{k}(s)=: \mathscr{H}_{l}^{1}(t)+\mathscr{H}_{l}^{0}(t), \quad l \in \mathscr{T} .
\end{aligned}
$$

First, we will prove that $\mathscr{H}_{l}^{1} \in \operatorname{SAP}\left(\mathbb{R}, L^{2}(P, \mathbb{Q})\right), l \in \mathscr{T}$. For any $\varepsilon>0$, since $\alpha_{l} \in \operatorname{SAP}\left(\mathbb{T}, \mathbb{R}^{+}\right), \quad F_{l}^{1}, G_{l k}^{1} \in \mathrm{SAP}$ $\left(\mathbb{R}, L^{2}(P, \mathbb{Q})\right)$, it is possible to find a real number $L=L(\varepsilon)>0$; for any interval with length $L(\varepsilon)$, there exists a number $\tau=\tau(\varepsilon)$ in this interval such that $\mathrm{E} \| \alpha_{l}(s+\tau)$ $-\alpha_{l}(s) \|^{2}<\varepsilon$ and

$$
\begin{gathered}
E\left\|F_{l}^{1}(s+\tau)-F_{l}^{1}(s)\right\|_{\mathbb{Q}}^{2}<\varepsilon, \\
E\left\|G_{l k}^{1}(s+\tau)-G_{l k}^{1}(s)\right\|_{\mathbb{Q}}^{2}<\varepsilon .
\end{gathered}
$$

For $l \in \mathscr{T}$, then we consider

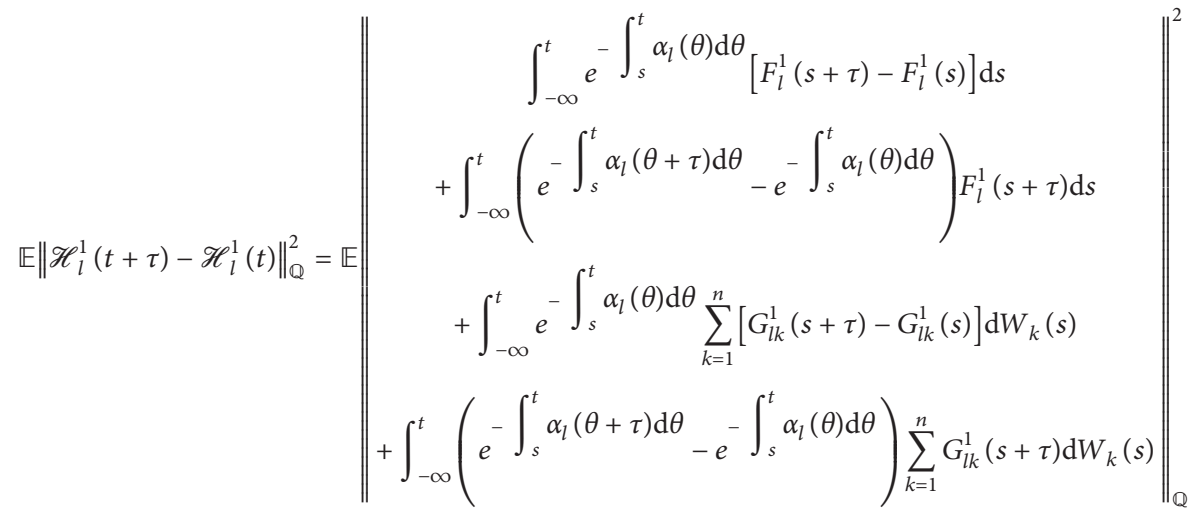

$$
\begin{aligned}
& \leq 4 \mathbb{E}\left\|\int_{-\infty}^{t} e^{-\int_{s}^{t} \alpha_{l}(\theta) \mathrm{d} \theta}\left[F_{l}^{1}(s+\tau)-F_{l}^{1}(s)\right] \mathrm{d} s\right\|_{\mathbb{Q}}^{2} \\
& +4 \mathbb{E}\left\|\int_{-\infty}^{t}\left(e^{-\int_{s}^{t} \alpha_{l}(\theta+\tau) \mathrm{d} \theta}-e^{-\int_{s}^{t} \alpha_{l}(\theta) \mathrm{d} \theta}\right) F_{l}^{1}(s+\tau) \mathrm{d} s\right\|_{\mathbb{Q}}^{2} \\
& +4 \mathbb{E}\left\|\int_{-\infty}^{t} e^{-\int_{s}^{t} \alpha_{l}(\theta) \mathrm{d} \theta} \sum_{k=1}^{n}\left[G_{l k}^{1}(s+\tau)-G_{l k}^{1}(s)\right] \mathrm{d} W_{k}(s)\right\|_{\mathbb{Q}}^{2} \\
& +4 \mathbb{E}\left\|\int_{-\infty}^{t}\left(e^{-\int_{s}^{t} \alpha_{l}(\theta+\tau) \mathrm{d} \theta}-e^{-\int_{s}^{t} \alpha_{l}(\theta) \mathrm{d} \theta}\right) \sum_{k=1}^{n} G_{l k}^{1}(s+\tau) \mathrm{d} W_{k}(s)\right\|_{\mathbb{Q}}^{2} \\
& \leq \frac{8}{\left(\alpha_{l}^{-}\right)^{2}}\left(\sum_{k=1}^{n}\left(b_{l k}^{1^{+}}\right)^{2} \sum_{k=1}^{n}\left(L_{l}^{f}\right)^{2}+\sum_{k=1}^{n}\left(c_{l k}^{1^{+}}\right)^{2} \sum_{k=1}^{n}\left(L_{l}^{g}\right)^{2}+\left(\mathscr{F}_{l}^{1^{+}}\right)^{2}+\frac{\alpha_{l}^{-}}{2} \sum_{k=1}^{n}\left(L_{l k}^{\delta}\right)^{2}+4\right) \varepsilon \text {. }
\end{aligned}
$$


Thus, we proved that $\mathscr{H}_{l}^{1}$ is square-mean almost periodic, $l \in \mathscr{T}$.

On the other hand, we will prove that $\mathscr{H}_{l}^{0} \in \operatorname{SPAP}_{0}\left(\mathbb{R}, L^{2}(P, \mathbb{Q})\right)$. Hence, we only need to show

$$
\lim _{T \longrightarrow+\infty} \frac{1}{2 T} \int_{-T}^{T} E\left\|\mathscr{H}_{l}^{0}(t)\right\|_{\mathbb{Q}}^{2} \mathrm{~d} t=0, \quad l \in \mathscr{T} .
$$

Thus, we obtain

$$
\begin{aligned}
\frac{1}{2 T} \int_{-T}^{T} E\left\|\mathscr{H}_{l}^{0}(t)\right\|_{\mathbb{Q}}^{2} \mathrm{~d} t & =\frac{1}{2 T} \int_{-T}^{T} E\left\|\int_{-\infty}^{t} e^{-\int_{s}^{t} \alpha_{l}(\theta) \mathrm{d} \theta} F_{l}^{0}(s) \mathrm{d} s+\int_{-\infty}^{t} e^{-\int_{s}^{t} \alpha_{l}(\theta) \mathrm{d} \theta} \sum_{k=1}^{n} G_{l k}^{0}(s) \mathrm{d} W_{k}(s)\right\|_{\mathbb{Q}}^{2} \mathrm{~d} t \\
& \leq \frac{1}{T} \int_{-T}^{T} E\left\|\int_{-\infty}^{t} e^{-\int_{s}^{t} \alpha_{l}(\theta) \mathrm{d} \theta} F_{l}^{0}(s) \mathrm{d} s\right\|_{\mathbb{Q}}^{2} \mathrm{~d} t+\frac{1}{T} \int_{-T}^{T} E\left\|\int_{-\infty}^{t} e^{-\int_{s}^{t} \alpha_{l}(\theta) \mathrm{d} \theta} \sum_{k=1}^{n} G_{l k}^{0}(s) \mathrm{d} W_{k}(s)\right\|_{\mathbb{Q}}^{2} \mathrm{~d} t=: \mathbb{Q}_{l}^{1}+\mathbb{Q}_{l}^{2} .
\end{aligned}
$$

It follows from the Cauchy-Schwarz inequality that

$$
\begin{aligned}
Q_{l}^{1} & =\frac{1}{T} \int_{-T}^{T} E\left\|\int_{-\infty}^{t} e^{-\int_{s}^{t} \alpha_{l}(\theta) \mathrm{d} \theta} F_{l}^{0}(s) \mathrm{d} s\right\|_{\mathbb{Q}}^{2} \mathrm{~d} t \\
& \leq \frac{1}{T} \int_{-T}^{T}\left[\int_{-\infty}^{t} e^{-\int_{s}^{t} \alpha_{l}(\theta) \mathrm{d} \theta} \mathrm{d} s\right]\left[\int_{-\infty}^{t} e^{-\int_{s}^{t} \alpha_{l}(\theta) \mathrm{d} \theta} E\left\|F_{l}^{0}(s)\right\|_{\mathbb{Q}}^{2} \mathrm{~d} s\right] \mathrm{d} t \\
& \leq \frac{1}{T \alpha_{l}^{-}} \int_{-T}^{T} \int_{-T}^{t} e^{-\int_{s}^{t} \alpha_{l}(\theta) \mathrm{d} \theta} E\left\|F_{l}^{0}(s)\right\|_{\mathbb{Q}}^{2} \mathrm{~d} s \mathrm{~d} t+\frac{1}{T \alpha_{l}^{-}} \int_{-T}^{T} \int_{-\infty}^{-T} e^{-\int_{s}^{t} \alpha_{l}(\theta) \mathrm{d} \theta} E\left\|F_{l}^{0}(s)\right\|_{\mathbb{Q}}^{2} \mathrm{~d} s \mathrm{~d} t=\Omega_{1}+\Omega_{2} .
\end{aligned}
$$

Set $\zeta=t-s$, and using Fubini's theorem, we obtain that

$$
\begin{aligned}
\Omega_{1} & =\frac{1}{T \alpha_{l}^{-}} \int_{-T}^{T} \int_{-T}^{t} e^{-\int_{s}^{t} \alpha_{l}(\theta) \mathrm{d} \theta} E\left\|F_{l}^{0}(s)\right\|_{\mathbb{Q}}^{2} \mathrm{~d} s \mathrm{~d} t \\
& \leq \frac{1}{T \alpha_{l}^{-}} \int_{-T}^{T} \int_{-T}^{t} e^{-(t-s) \alpha_{l}^{-}} E\left\|F_{l}^{0}(s)\right\|_{Q^{2}}^{2} \mathrm{~d} s \mathrm{~d} t \\
& \leq \frac{1}{T \alpha_{l}^{-}} \int_{-T}^{T} \int_{0}^{+\infty} e^{-\zeta \alpha_{l}^{-}} E\left\|F_{l}^{0}(t-\zeta)\right\|_{Q^{2}} \mathrm{~d} \zeta \mathrm{d} t \\
& \leq \frac{2}{\alpha_{l}^{-}} \int_{0}^{+\infty} e^{-\zeta \alpha_{l}^{-}}\left[\frac{T+\zeta}{T} \frac{1}{2(T+\zeta)} \int_{-T-\zeta}^{T-\zeta} E\left\|F_{l}^{0}(\theta)\right\|_{\mathbb{Q}}^{2} \mathrm{~d} \theta\right] \mathrm{d} \zeta \\
& \leq \frac{2}{\left(\alpha_{l}^{-}\right)^{2}}\left[\frac{1}{2 T} \int_{-T}^{T} E\left\|F_{l}^{0}(s)\right\|_{Q^{2}} \mathrm{~d} s\right],
\end{aligned}
$$

since $\quad F_{l}^{0} \in \operatorname{SPAP}_{0}\left(\mathbb{R}, L^{2}(P, \mathbb{Q})\right)$, thus $\quad \Omega_{1} \longrightarrow 0 \quad$ as $T \longrightarrow+\infty$. Since $F_{l}^{0}$ is bounded, as $T \longrightarrow+\infty$, we have

$$
\begin{aligned}
\Omega_{2} & =\frac{1}{T \alpha_{l}^{-}} \int_{-T}^{T} \int_{-\infty}^{-T} e^{-\int_{s}^{t} \alpha_{l}(\theta) \mathrm{d} \theta} E\left\|F_{l}^{0}(s)\right\|_{\mathbb{Q}}^{2} \mathrm{~d} s \mathrm{~d} t \\
& \leq \frac{1}{T \alpha_{l}^{-}} \int_{-T}^{T} \int_{T+t}^{+\infty} e^{-\zeta \alpha_{l}^{-}} E\left\|F_{l}^{0}(t-\zeta)\right\|_{\mathbb{Q}}^{2} \mathrm{~d} \zeta \mathrm{d} t \\
& \leq \frac{1}{T\left(\alpha_{l}^{-}\right)^{3}} \sup _{t \in \mathbb{R}} E\left\|F_{l}^{0}(t)\right\|_{\mathbb{Q}}^{2} \int_{-T}^{T} \int_{T+t}^{+\infty} e^{-\zeta \alpha_{l}^{-}} \mathrm{d} \zeta \mathrm{d} t \\
& =\frac{\left[1-e^{-2 \alpha_{l}^{-} T}\right]}{T\left(\alpha_{l}^{-}\right)^{3}} \sup _{t \in \mathbb{R}} E\left\|F_{l}^{0}(t)\right\|_{\mathbb{Q}}^{2} \longrightarrow 0 .
\end{aligned}
$$

Hence, for $l \in \mathscr{T}$, we have that $\mathbb{Q}_{l}^{1} \longrightarrow 0$ as $T \longrightarrow+\infty$. By the Itô isometry, one has 


$$
\begin{aligned}
Q_{l}^{2}= & \frac{1}{T} \int_{-T}^{T} E\left\|\int_{-\infty}^{t} e^{-\int_{s}^{t} \alpha_{l}(\theta) \mathrm{d} \theta} \sum_{k=1}^{n} G_{l k}(s) \mathrm{d} W_{k}(s)\right\|_{\mathbb{Q}}^{2} \mathrm{~d} t \\
\leq & \frac{1}{T} \sum_{k=1}^{n} \int_{-T}^{T} \int_{-T}^{t} e^{-2 \alpha_{l}^{-}(t-s)} E\left\|G_{l k}(s)\right\|_{\mathbb{Q}}^{2} \mathrm{~d} s \mathrm{~d} t \\
& +\frac{1}{T} \sum_{k=1}^{n} \int_{-T}^{T} \int_{-\infty}^{-T} e^{-2 \alpha_{l}^{-}(t-s)} E\left\|G_{l k}(s)\right\|_{\mathbb{Q}}^{2} \mathrm{~d} s \mathrm{~d} t \\
\leq & \frac{1}{\alpha_{l}^{-}} \sum_{k=1}^{n}\left[\frac{1}{2 T} \int_{-T}^{T} E\left\|G_{l k}(s)\right\|_{\mathbb{Q}}^{2} \mathrm{~d} s\right] \\
& +\frac{\left[1-e^{-4 \alpha_{l}^{-} T}\right]}{T\left(\alpha_{l}^{-}\right)^{2}} \sup _{t \in \mathbb{R}} \sum_{k=1}^{n} E\left\|G_{l k}(t)\right\|_{\mathbb{Q}}^{2} \longrightarrow 0, \quad \text { as } T \longrightarrow+\infty .
\end{aligned}
$$

Therefore, we have verified

$$
\lim _{T \longrightarrow+\infty} \frac{1}{2 T} \int_{-T}^{T} E\left\|\mathscr{H}_{l}^{0}(t)\right\|_{\mathbb{Q}}^{2} \mathrm{~d} t=0, \quad l \in \mathscr{T} .
$$

Therefore, we have $u_{l}^{\phi} \in \operatorname{SPAP}\left(\mathbb{R}, L^{2}(P, \mathbb{Q})\right)$.

Next, we show that $\Phi$ is a self-mapping from $\mathscr{X}^{*}$ to $\mathscr{X}^{*}$. For $\phi \in \mathscr{X}^{*}$, by the Cauchy-Schwarz inequality and Itô isometry, we have

$$
\begin{aligned}
& E\left\|(\Phi \phi)(t)-\phi^{0}(t)\right\|_{\mathbb{Q}^{n}}^{2}=\max _{l \in \mathscr{T}}\left\{E\left\|\int_{-\infty}^{t} e^{-\int_{s}^{t} \alpha_{l}(\theta) \mathrm{d} \theta}\left[\sum_{k=1}^{n} b_{l k}(s) f_{k}\left(\phi_{k}(s)\right)+\sum_{k=1}^{n} c_{l k}(s) g_{k}\left(\phi_{k}\left(s-v_{k l}(s)\right)\right)\right] \mathrm{d} s+\int_{-\infty}^{t} e^{-\int_{s}^{t} \alpha_{l}(\theta) \mathrm{d} \theta} \sum_{k=1}^{n} \delta_{l k}\left(\phi_{k}\left(s-\eta_{l k}(s)\right)\right) \mathrm{d} W_{k}(s)\right\|_{\mathbb{Q}}^{2}\right\} \\
& \leq 3 \max _{l \in \mathscr{T}}\left\{E\left\|\int_{-\infty}^{t} e^{-\int_{s}^{t} \alpha_{l}(\theta) \mathrm{d} \theta} \sum_{k=1}^{n} b_{l k}(s) f_{k}\left(\phi_{k}(s)\right) \mathrm{d} s\right\|_{\mathbb{Q}}^{2}\right\} \\
& +3 \max _{l \in \mathscr{T}}\left\{E\left\|\int_{-\infty}^{t} e^{-\int_{s}^{t} \alpha_{l}(\theta) \mathrm{d} \theta} \sum_{k=1}^{n} c_{l k}(s) g_{k}\left(\phi_{k}\left(s-v_{k l}(s)\right)\right) \mathrm{d} s\right\|_{\mathbb{Q}}^{2}\right\} \\
& +3 \max _{l \in \mathscr{T}}\left\{E\left\|\int_{-\infty}^{t} e^{-\int_{s}^{t} \alpha_{l}(\theta) \mathrm{d} \theta} \sum_{k=1}^{n} \delta_{l k}\left(\phi_{k}\left(s-\eta_{l k}(s)\right)\right) \mathrm{d} W_{k}(s)\right\|_{\mathbb{Q}}^{2}\right\} \\
& \leq \max _{l \in \mathscr{T}}\left\{\frac{3}{\alpha_{l}^{-}} E\left[\int_{-\infty}^{t} e^{-\int_{s}^{t} \alpha_{l}(\theta) \mathrm{d} \theta}\left(\sum_{k=1}^{n}\left\|b_{l k}(s)\right\|_{\mathbb{Q}} L_{k}^{f}\left\|\phi_{k}(s)\right\|_{\mathbb{Q}}\right)^{2} \mathrm{~d} s\right]\right\} \\
& +\max _{l \in \mathscr{T}}\left\{\frac{3}{\alpha_{l}^{-}} E\left[\int_{-\infty}^{t} e^{-\int_{s}^{t} \alpha_{l}(\theta) \mathrm{d} \theta}\left(\sum_{k=1}^{n}\left\|c_{l k}(s)\right\|_{\mathbb{Q}} L_{k}^{g}\left\|\phi_{k}\left(s-v_{k l}(s)\right)\right\|_{\mathbb{Q}}\right)^{2} \mathrm{~d} s\right]\right\} \\
& +3 \max _{l \in \mathscr{T}}\left\{E \sum_{k=1}^{n}\left(\int_{-\infty}^{t} e^{-2 \int_{s}^{t} \alpha_{l}(\theta) \mathrm{d} \theta}\left\|\delta_{l k}\left(\phi_{k}\left(s-\eta_{l k}(s)\right)\right)\right\|_{\mathbb{Q}}^{2} \mathrm{~d} s\right)\right\} \\
& \leq \max _{l \in \mathscr{T}}\left\{\frac{3}{\left(\alpha_{l}^{-}\right)^{2}}\left[\sum_{k=1}^{n}\left(\left\|b_{l k}(t)\right\|_{\mathbb{Q}}\right)^{2} \sum_{k=1}^{n}\left(L_{k}^{f}\right)^{2}\right]\right\} E\|\phi(t)\|_{\mathbb{Q}^{n}}^{2} \\
& +\max _{l \in \mathscr{T}}\left\{\frac{3}{\left(\alpha_{l}^{-}\right)^{2}}\left[\sum_{k=1}^{n}\left(K_{l k}^{*}\left\|c_{l k}(t)\right\|_{\mathbb{Q}}\right)^{2} \sum_{k=1}^{n}\left(L_{k}^{g}\right)^{2}\right]\right\} E\|\phi(t)\|_{\mathbb{Q}^{n}}^{2} \\
& +\max _{l \in \mathscr{T}}\left\{\frac{3}{2 \alpha_{l}^{-}} \sum_{k=1}^{n}\left(L_{l k}^{\delta}\right)^{2}\right\} E\|\phi(t)\|_{\mathbb{Q}^{n}}^{2} \\
& \leq \max _{l \in \mathscr{T}}\left\{\frac{3}{\left(\alpha_{l}^{-}\right)^{2}}\left[\sum_{k=1}^{n}\left(\left(\left\|b_{l k}(t)\right\|_{\mathbb{Q}}\right)^{2} \sum_{k=1}^{n}\left(L_{k}^{f}\right)^{2}+\sum_{k=1}^{n}\left(\left\|c_{l k}(t)\right\|_{\mathbb{Q}}\right)^{2} \sum_{k=1}^{n}\left(L_{k}^{g}\right)^{2}+\frac{\alpha_{l}^{-}}{2} \sum_{k=1}^{n}\left(L_{l k}^{\delta}\right)^{2}\right)\right]\right\} \\
& E\|\phi(t)\|_{\mathbb{Q}^{n}}^{2} .
\end{aligned}
$$


It follows from $(29)$ and $\left(H_{3}\right)$ that

$$
\begin{aligned}
\left\|\Phi \phi-\phi^{0}\right\|_{\mathscr{X}}^{2} & =\sup _{t \in \mathbb{R}} E\left\|(\Phi \phi)(t)-\phi^{0}(t)\right\|_{\mathbb{Q}^{n}}^{2} \\
& \leq \max _{l \in \mathscr{T}}\left\{\frac{3}{\left(\alpha_{l}^{-}\right)^{2}}\left[\sum_{k=1}^{n}\left(b_{l k}^{+}\right)^{2} \sum_{k=1}^{n}\left(L_{k}^{f}\right)^{2}+\sum_{k=1}^{n}\left(c_{l k}^{+}\right)^{2} \sum_{k=1}^{n}\left(L_{k}^{g}\right)^{2}+\frac{\alpha_{l}^{-}}{2} \sum_{k=1}^{n}\left(L_{l k}^{\delta}\right)^{2}\right]\right\} 4 \kappa^{2},
\end{aligned}
$$

which implies that $\left\|\Phi \phi-\phi^{0}\right\|_{\mathscr{X}} \leq \kappa$; hence, we have $\Phi \phi \in \mathscr{X}^{*}$. Next, we show that $\Phi$ is a contraction mapping in $\mathscr{X}^{*}$. For any $\phi, \psi \in \mathscr{X}^{*}$, we have

$$
\begin{aligned}
& E\|(\Phi \phi)(t)-(\Phi \psi)(t)\|_{\mathbb{Q}^{n}}^{2} \\
& \quad=\max _{l \in \mathscr{T}}\left\{\begin{array}{l}
E \int_{-\infty}^{t} e^{-\int_{s}^{t} \alpha_{l}(\theta) \mathrm{d} \theta}\left(\sum_{k=1}^{n} b_{l k}(s)\left[f_{k}\left(\phi_{k}(s)\right)-f_{k}\left(\psi_{k}(s)\right)\right]\right) \mathrm{d} s \\
+\int_{-\infty}^{t} e^{-\int_{s}^{t} \alpha_{l}(\theta) \mathrm{d} \theta}\left(\sum_{k=1}^{n} c_{l k}(s)\left[g_{k}\left(\phi_{k}\left(s-v_{k l}(s)\right)\right)-g_{k}\left(\psi_{k}\left(s-v_{k l}(s)\right)\right)\right]\right) \mathrm{d} s \\
+\int_{-\infty}^{t} e^{-\int_{s}^{t} \alpha_{l}(\theta) \mathrm{d} \theta} \sum_{k=1}^{n}\left[\delta_{l k}\left(\phi_{k}\left(s-\eta_{l k}(s)\right)\right)-\delta_{l k}\left(\psi_{k}\left(s-\eta_{l k}(s)\right)\right)\right] \mathrm{d} W_{k}(s)
\end{array}\right. \\
& \quad \leq \max _{l \in \mathscr{T}}\left\{\frac{3}{\left(\alpha_{l}^{-}\right)^{2}}\left[\sum_{k=1}^{n}\left(b_{l k}^{+}\right)^{2} \sum_{k=1}^{n}\left(L_{k}^{f}\right)^{2}+\sum_{k=1}^{n}\left(c_{l k}^{+}\right)^{2} \sum_{k=1}^{n}\left(L_{k}^{g}\right)^{2}+\frac{\alpha_{l}^{-}}{2} \sum_{k=1}^{n}\left(L_{l k}^{\delta}\right)^{2}\right]\|\phi(t)-\psi(t)\|_{\mathbb{Q}^{n} .}^{2} .\right.
\end{aligned}
$$

Thus, we have

$$
\sup _{t \in \mathbb{R}} E\|(\Phi \phi)(t)-(\Phi \psi)(t)\|_{\mathbb{Q}^{n}}^{2} \leq \max _{l \in \mathscr{T}}\left\{\frac{3 Y_{l}}{\left(\alpha_{l}^{-}\right)^{2}}\right\} \sup _{t \in \mathbb{R}} E\|\phi(t)-\psi(t)\|_{\mathbb{Q}^{n}}^{2}
$$

It follows from (32) that

$$
\|\Phi \phi-\Phi \psi\|_{\mathscr{X}} \leq \sqrt{\rho}\|\phi-\psi\|_{\mathscr{X}} .
$$

In view of $\left(\mathrm{H}_{3}\right)$, we see that $\Phi$ is a contraction mapping. Therefore, $\Phi$ has a unique fixed point in $\mathscr{X}^{*}$, that is, (1) has a unique square-mean pseudo almost periodic solution in $\mathscr{X}^{*}$. The proof is finished.

\section{Square-Mean Exponential Stability}

In this section, we will consider the square-mean exponential stability of system (1).

Definition 4. Let $u=\left(u_{1}, u_{2}, \ldots, u_{n}\right)^{T}$ be a solution of (1) with the initial value $\phi=\left(\phi_{1}, \phi_{2}, \ldots, \phi_{n}\right)^{T}$. If there exist positive constants $\lambda>0$ and $\mathscr{M}>0$ such that for an arbitrary solution $w=\left(w_{1}, w_{2}, \ldots, w_{n}\right)^{T}$ with initial value, $\psi=\left(\psi_{1}, \psi_{2}, \ldots, \psi_{n}\right)^{T}$ satisfies

$$
E\|w(t)-u(t)\|_{\mathbb{Q}^{n}}^{2} \leq \mathscr{M} e^{-\lambda t} E\|\psi-\phi\|_{1}^{2}, \quad t>0,
$$

where

$$
\begin{aligned}
\|w(t)-u(t)\|_{\mathbb{Q}^{n}} & =\max _{l \in \mathscr{T}}\left\{\left\|w_{l}(t)-u_{l}(t)\right\|_{\mathbb{Q}}\right\}, \\
\|\psi-\phi\|_{1} & =\max _{l \in \mathscr{T}}\left\{\sup _{s \in[-v, 0]}\left\|\psi_{l}(s)-\phi_{l}(s)\right\|_{\mathbb{Q}}\right\} .
\end{aligned}
$$

Then, the solution $u(t)$ is said to be square-mean exponentially stable. 
Theorem 2. Suppose that the assumptions $\left(H_{1}\right)-\left(H_{4}\right)$ are satisfied, then for any initial value of dynamical system (1), there exists a square-mean pseudo almost periodic solution $u(t)$, which is square-mean exponentially stable.

Proof. From Theorem 1, we see that system (1) has a squaremean pseudo almost periodic solution $u=\left(u_{1}, u_{2}, \ldots, u_{n}\right)^{T}$ with initial value $\phi=\left(\phi_{1}, \phi_{2}, \ldots, \phi_{n}\right)^{T}$. Suppose that $w=$ $\left(w_{1}, w_{2}, \ldots, w_{n}\right)^{T}$ is an arbitrary solution of system (1) with initial value $\psi=\left(\psi_{1}, \psi_{2}, \ldots, \psi_{n}\right)^{T}$ and $z(t)=w(t)-u(t)$. Let $\delta(t)=\left(\delta_{l k}(t)\right)_{n \times n}$, where $\delta_{l k}(t)=\delta_{l k}\left(z_{k}\left(t-\eta_{l k}(t)\right)\right)$. We consider the Lyapunov function as follows:

$$
V(t, z(t))=\max _{l \in \mathscr{T}}\left\{e^{\lambda t} \xi_{l} z_{l}^{*}(t) z_{l}(t)+\xi_{l} \Gamma_{l}(t, z(t))\right\}
$$

where

$$
\Gamma_{l}(t, z(t))=\sum_{k=1}^{n}\left(c_{l k}^{+}\right)^{2}\left(L_{k}^{g}\right)^{2} \frac{e^{\lambda v^{+}}}{1-\gamma} \int_{t-v_{k l}(t)}^{t} z_{k}^{*}(s) z_{k}(s) e^{\lambda s} \mathrm{~d} s+\sum_{k=1}^{n}\left(L_{l k}^{\delta}\right)^{2} \frac{e^{\lambda \eta^{+}}}{1-\beta} \int_{t-\eta_{l k}(t)}^{t} z_{k}^{*}(s) z_{k}(s) e^{\lambda s} \mathrm{~d} s
$$

Then, by Itô formula, we have the following stochastic differential:

$$
\mathrm{d} V(t, z(t))=\mathscr{L} V(t, z(t)) \mathrm{d} t+V_{z}(t, z(t)) \delta(t) \mathrm{d} W(t)
$$

where $V_{z}(t, z(t))=\left(\partial V(t, z(t)) / \partial z_{1}, t \ldots n, q \partial V(t, z(t)) /\right.$ $\left.\partial z_{n}\right)$, and $\mathscr{L}$ is the weak infinitesimal operator such that

$$
\begin{aligned}
& \mathscr{L} V(t, z(t))=\max _{l \in \Phi}\left\{\begin{array}{c}
\lambda e^{\lambda t} \xi_{l} z_{l}^{*}(t) z_{l}(t)+e^{\lambda t} \xi_{l} z_{l}(t)\left[-\alpha_{l}(t) z_{l}^{*}(t)+\sum_{k=1}^{n}\left(b_{l k}(t)\left[f_{k}\left(w_{k}(t)\right)-f_{k}\left(u_{k}(t)\right)\right]\right)^{*}+\sum_{k=1}^{n}\left(c_{l k}(t)\left[g_{k}\left(w_{k}\left(t-v_{k l}(t)\right)\right)-g_{k}\left(u_{k}\left(t-v_{k l}(t)\right)\right)\right]\right)^{*}\right] \\
+e^{\lambda t} \xi_{l} z_{l}^{*}(t)\left[-\alpha_{l}(t) z_{l}(t)+\sum_{k=1}^{n} b_{l k}(t)\left[f_{k}\left(w_{k}(t)\right)-f_{k}\left(u_{k}(t)\right)\right]+\sum_{k=1}^{n} c_{l k}(t)\left[g_{k}\left(w_{k}\left(t-v_{k l}(t)\right)\right)-g_{k}\left(u_{k}\left(t-v_{k l}(t)\right)\right)\right]\right] \\
+\xi_{l} \sum_{k=1}^{n}\left(c_{l k}^{+}\right)^{2}\left(L_{k}^{g}\right)^{2} \frac{e^{\lambda v^{+}}}{1-\gamma} z_{k}^{*}(t) z_{k}(t) e^{\lambda t}-\xi_{l} \sum_{k=1}^{n}\left(c_{l k}^{+}\right)^{2}\left(L_{k}^{g}\right)^{2} \frac{e^{\lambda v^{+}}}{1-\gamma}\left(1-v_{k l}^{\prime}(t)\right) \\
\times z_{k}^{*}\left(t-v_{k l}(t)\right) z_{k}\left(t-v_{k l}(t)\right) e^{\lambda\left(t-v_{k l}(t)\right)} \mathrm{d} s+\xi_{l} \sum_{k=1}^{n}\left(L_{l k}^{\delta}\right)^{2} \frac{e^{\lambda \eta^{+}}}{1-\beta^{+}} z_{k}^{*}(t) z_{k}(t) e^{\lambda t} \\
-\xi_{l} \sum_{k=1}^{n}\left(L_{l k}^{\delta}\right)^{2} \frac{e^{\lambda \eta^{+}}}{1-\beta}\left(1-\eta_{l k}^{\prime}(t)\right) z_{k}^{*}\left(t-\eta_{l k}(t)\right) z_{k}\left(t-\eta_{l k}(t)\right) e^{\lambda\left(t-\eta_{l k}(t)\right)} \\
+e^{\lambda t} \xi_{l} \sum_{k=1}^{n}\left[\delta_{l k}\left(w_{k}\left(t-\eta_{l k}(t)\right)\right)-\delta_{l k}\left(u_{k}\left(t-\eta_{l k}(t)\right)\right)\right]^{*}\left[\delta_{l k}\left(w_{k}\left(t-\eta_{l k}(t)\right)\right)-\delta_{l k}\left(u_{k}\left(t-\eta_{l k}(t)\right)\right)\right]
\end{array}\right\} \\
& \leq \max _{l \in J}\left\{\begin{array}{c}
e^{\lambda t}\left(\lambda \xi_{l}-2 \xi_{l} \alpha_{l}(t)\right) z_{l}^{*}(t) z_{l}(t)+e^{\lambda t} \xi_{l} \sum_{k=1}^{n}\left(b_{l k}(t)\left[f_{k}\left(w_{k}(t)\right)-f_{k}\left(u_{k}(t)\right)\right]\right)^{*} \\
\times\left(b_{l k}(t)\left[f_{k}\left(w_{k}(t)\right)-f_{k}\left(u_{k}(t)\right)\right]\right)+e^{\lambda t} \xi_{l} \sum_{k=1}^{n}\left(c_{l k}(t)\left[g_{k}\left(w_{k}\left(t-v_{k l}(t)\right)\right)-g_{k}\left(u_{k}\left(t-v_{k l}(t)\right)\right)\right]\right)^{*} \\
\left(c_{l k}(t)\left[g_{k}\left(w_{k}\left(t-v_{k l}(t)\right)\right)-g_{k}\left(u_{k}\left(t-v_{k l}(t)\right)\right)\right]\right) \\
+2 e^{\lambda t} \xi_{l} z_{l}^{*}(t) z_{l}(t)+e^{\lambda t} \sum_{p=1}^{n} \xi_{l} \sum_{k=1}^{n}\left(c_{l k}^{+}\right)^{2}\left(L_{k}^{g}\right)^{2} \frac{e^{\lambda v^{+}}}{1-\gamma} z_{k}^{*}(t) z_{k}(t)-e^{\lambda t} \xi_{l} \sum_{k=1}^{n}\left(c_{l k}^{+}\right)^{2} \\
x\left(L_{k}^{g}\right)^{2} \frac{e^{\lambda v^{+}}}{1-\gamma}(1-\gamma) z_{k}^{*}\left(t-v_{k l}(t)\right) z_{k}\left(t-v_{k l}(t)\right) e^{\lambda v^{+}}+e^{\lambda t} \xi_{l} \sum_{k=1}^{n}\left(L_{l k}^{\delta}\right)^{2} \frac{e^{\lambda \eta^{+}}}{1-\beta^{+}} z_{k}^{*}(t) z_{k}(t) \\
-e^{\lambda t} \xi_{l} \sum_{k=1}^{n}\left(L_{l k}^{\delta}\right)^{2} \frac{e^{\lambda \eta^{+}}}{1-\beta}(1-\beta) z_{k}^{*}\left(t-\eta_{l k}(t)\right) \times z_{k}\left(t-\eta_{l k}(t)\right) e^{-\lambda \eta^{+}} \\
+e^{\lambda t} \xi_{l} \sum_{k=1}^{n}\left(L_{l k}^{\delta}\right)^{2} z_{k}^{*}\left(t-\eta_{l k}(t)\right) z_{k}\left(t-\eta_{l k}(t)\right)
\end{array}\right\}
\end{aligned}
$$




$$
\begin{aligned}
& \leq \max _{l \in \mathscr{T}}\left\{\begin{array}{c}
e^{\lambda t}\left(\lambda \xi_{l}+2 \xi_{l}-2 \xi_{l} \alpha_{l}^{-}\right) z_{l}^{*}(t) z_{l}(t)+e^{\lambda t} \xi_{l} \sum_{k=1}^{n}\left(b_{l k}^{+}\right)^{2}\left(L_{k}^{f}\right)^{2} z_{k}^{*}(t) z_{k}(t) \\
+e^{\lambda t} \xi_{l} \sum_{k=1}^{n}\left(c_{l k}^{+}\right)^{2}\left(L_{k}^{g}\right)^{2} \frac{e^{\lambda v^{+}}}{1-\gamma} z_{k}^{*}(t) z_{k}(t)+e^{\lambda t} \xi_{l} \sum_{k=1}^{n}\left(L_{l k}^{\delta}\right)^{2} \frac{e^{\lambda \eta^{+}}}{1-\beta} z_{k}^{*}(t) z_{k}(t)
\end{array}\right\} \\
& =\max _{l \in \mathscr{T}}\left\{e^{\lambda t}\left\{\left(\lambda \xi_{l}+2 \xi_{l}-2 \xi_{l} \alpha_{l}^{-}+\sum_{k=1}^{n} \xi_{k}\left(b_{k l}^{+}\right)^{2}\left(L_{k}^{f}\right)^{2}+\sum_{k=1}^{n} \xi_{k}\left(c_{k l}^{+}\right)^{2}\left(L_{k}^{g}\right)^{2} \frac{e^{\lambda v^{+}}}{1-\gamma}+\sum_{k=1}^{n} \xi_{k}\left(L_{k l}^{\delta}\right)^{2} \frac{e^{\lambda \eta^{+}}}{1-\beta}\right)\right\} z_{l}^{*}(t) z_{l}(t)\right\} .
\end{aligned}
$$

From $\left(H_{4}\right)$, we easily derive

$$
\mathscr{L} V(t, z(t)) \leq 0 .
$$

Now, similar to the previous literature, we define the stopping time (or Markov time) $\sigma_{k}:=\inf \{s \geq 0:|z(s)| \geq k\}$, and by the Dynkin formula, we have

$$
E\left[V\left(t \wedge \sigma_{k}, z\left(t \wedge \sigma_{k}\right)\right)\right]=E\left[V(0, z(0)]+E\left[\int_{0}^{t \wedge \sigma_{k}} \mathscr{L} V(s, z(s)) \mathrm{d} s\right]\right.
$$

Letting $k \longrightarrow \infty$ on both sides (41), from the monotone convergence theorem, we can get

$$
\begin{aligned}
E[V(t, z(t))] \leq & E[V(0, z(0)] \\
= & \max _{l \in \mathscr{T}}\left\{\xi_{l} E z_{l}^{*}(0) z_{l}(0)+\xi_{l} \sum_{k=1}^{n}\left(c_{l k}^{+}\right)^{2}\left(L_{k}^{g}\right)^{2} \times \frac{e^{\lambda v^{+}}}{1-\gamma} \int_{-v_{k l}(0)}^{0} E z_{k}^{*}(s) z_{k}(s) e^{\lambda s} \mathrm{~d} s\right. \\
& \left.+\xi_{l} \sum_{k=1}^{n}\left(L_{l k}^{\delta}\right)^{2} \frac{e^{\lambda \eta^{+}}}{1-\beta} \int_{-\eta_{l k}(0)}^{0} E z_{k}^{*}(s) z_{k}(s) e^{\lambda s} \mathrm{~d} s\right\} \\
\leq & \max _{l \in \mathscr{T}}\left\{\left(\xi_{l}+\xi_{l} \sum_{k=1}^{n}\left(b_{k l}^{+}\right)^{2}\left(L_{k}^{g}\right)^{2} v^{+} \frac{e^{\lambda v^{+}}}{1-\gamma}+\xi_{l} \sum_{k=1}^{n}\left(L_{k l}^{\delta}\right)^{2} \eta^{+} \frac{e^{\lambda \eta^{+}}}{1-\beta}\right) E \sup _{s \in[-\gamma, 0]}\left\|\psi_{l}(s)-\phi_{l}(s)\right\|_{\mathbb{Q}}^{2}\right\} .
\end{aligned}
$$

On the other hand, it follows from the definition of $V(t, z(t))$ that

$E[V(t, z(t))] \geq E e^{\lambda t} \max _{l \in \mathscr{T}} \xi_{l} z_{l}^{*}(t) z_{l}(t) \geq e^{\lambda t} \min _{l \in \mathscr{T}} \xi_{l} E\|z(t)\|_{\mathbb{Q}^{n}}^{2}$
Combining (42) and (43), the following holds:

$$
E\|z(t)\|_{\mathbb{Q}^{n}}^{2} \leq \mathscr{M} e^{-\lambda t} E\|\psi-\phi\|_{1}^{2},
$$

where

$$
\mathscr{M}=\frac{1}{\min _{l \in \mathscr{T}} \xi_{l}} \max _{l \in \mathscr{T}} \xi_{l}\left\{1+\sum_{k=1}^{n}\left(c_{k l}^{+}\right)^{2}\left(L_{k}^{g}\right)^{2} v^{+} \frac{e^{\lambda v^{+}}}{1-\gamma}+\sum_{k=1}^{n}\left(L_{k l}^{\delta}\right)^{2} \eta^{+} \frac{e^{\lambda \eta^{+}}}{1-\beta}\right\},
$$

which together with Definition 4 verifies that square-mean pseudo almost periodic solution of system (1) which is square-mean exponentially stable. The proof is finished.
Remark 2. From the proofs of Theorems 1 and 2, one can easily see that if all the coefficients of (1) are square-mean almost automorphic and square-mean almost periodic, 
respectively, then, similar to the proofs of Theorems 1 and 2 and under the same corresponding conditions, one can show that the similar results of Theorems 1 and 2 are still valid for both cases of the square-mean pseudo almost automorphy and square-mean weighted pseudo almost periodicity.

\section{Illustrative Example}

In this section, we give numerical examples to illustrate the feasibility and effectiveness of our results obtained in Sections 3 and 4.

Example 1. Let $n=2$. Consider the following quaternionvalued stochastic neural network:

$$
\begin{aligned}
\mathrm{d} u_{l}(t)= & {\left[-\alpha_{l}(t) u_{l}(t)+\sum_{k=1}^{2} b_{l k}(t) f_{k}\left(u_{k}(t)\right)+\sum_{k=1}^{2} c_{l k}(t) g_{k}\left(u_{k}\left(t-v_{l k}(t)\right)+\mathscr{I}_{l}(t)\right] \mathrm{d} t\right.} \\
& +\sum_{k=1}^{2} \delta_{l k}\left(u_{k}\left(t-\eta_{l k}(t)\right)\right) \mathrm{d} W_{k}(t),
\end{aligned}
$$

where $l=1,2$; the coefficients are as follows:

$$
\begin{aligned}
f_{k}\left(u_{k}\right) & =g_{k}\left(u_{k}\right)=\frac{1}{7} \tan h u_{k}^{R}+i \frac{1}{5}\left|u_{k}^{I}\right|+j \frac{1}{4} \sin u_{k}^{I}+k \frac{1}{9} \sin \left(u_{k}^{J}+u_{k}^{K}\right), \\
\delta_{l k}\left(u_{k}\right) & =\frac{1}{12} \sin \left(u_{k}^{R}+u_{k}^{I}\right)+i \frac{1}{8} \sin u_{k}^{I}+j \frac{1}{9}\left|u_{k}^{J}\right|+k \frac{1}{5} \tan h u_{k}^{K}, \\
\alpha_{1}(t) & =3+|\cos (\sqrt{2} t)|, \\
\alpha_{2}(t) & =6-2 \sin (\sqrt{3} t), \\
b_{l k}(t) & =0.5 \sin (\sqrt{2} t)+i 0.5 \sin t+j 0.8 \cos t+k 0.4 \cos (2 t), \\
c_{l k}(t) & =0.8 \cos t+i 1.2 \sin (2 t)+j 1.5 \sin t+k 0.9 \cos (\sqrt{3} t), \\
\mathscr{I}_{1}(t) & =0.2 \sin (\sqrt{3} t)+i 0.5 \cos (2 t)+j 0.3 \cos (\sqrt{2} t)+k 0.3 \sin (\sqrt{(3)} t), \\
\mathscr{I}_{2}(t) & =0.3 \cos (\sqrt{2} t)+i 0.4 \sin (\sqrt{3} t)+j 0.5 \sin t+k 0.2 \cos (\sqrt{3} t)), \\
v_{k l}(t) & =\frac{1}{2}|\sin t|, \\
\eta_{l k}(t) & =\frac{4}{5}|\cos t| .
\end{aligned}
$$

Through simple calculations, we have 


$$
\begin{aligned}
\alpha_{1}^{-} & =3, \\
\alpha_{2}^{-} & =4, \\
L_{k}^{f} & =L_{k}^{g}=\frac{1}{4}, \\
L_{l k}^{\delta} & =\frac{1}{5}, \\
v^{+} & =\frac{1}{2}, \\
\eta^{+} & =\frac{4}{5}, \\
\gamma & =\frac{1}{2}, \\
\beta & =\frac{4}{5}, \\
b_{l k}^{+} & \leq 1.1402, \\
c_{l k}^{+} \leq 2.2672, & \approx 1.7701 . \\
Y_{1} & \approx 1.6901, \\
Y_{2} & \approx 1.7
\end{aligned}
$$

Then, we have

$$
\max \left\{\frac{3 Y_{1}}{\left(\alpha_{1}^{-}\right)^{2}}, \frac{3 Y_{2}}{\left(\alpha_{2}^{-}\right)^{2}}\right\} \approx \max \{0.5634,0.3319\}=0.5634=\rho<1 .
$$

Take $\lambda=0.1, \xi_{1}=0.3$, and $\xi_{2}=0.4$, then we have

$$
\begin{aligned}
2 \xi_{1} \alpha_{1}^{-}= & 1.8>\lambda \xi_{1}+2 \xi_{1}+\sum_{k=1}^{2} \xi_{k}\left(b_{k 1}^{+}\right)^{2}\left(L_{k}^{f}\right)^{2} \\
& +\sum_{k=1}^{2} \xi_{k}\left(c_{k 1}^{+}\right)^{2}\left(L_{k}^{g}\right)^{2} \frac{e^{\lambda v^{+}}}{1-\gamma}+\sum_{k=1}^{2} \xi_{k}\left(L_{k 1}^{\delta}\right)^{2} \frac{e^{\lambda \eta^{+}}}{1-\beta} \approx 0.9501, \\
2 \xi_{2} \alpha_{2}^{-}= & 3.2>\lambda \xi_{2}+2 \xi_{2}+\sum_{k=1}^{2} \xi_{k}\left(b_{k 2}^{+}\right)^{2}\left(L_{k}^{f}\right)^{2} \\
& +\sum_{k=1}^{2} \xi_{k}\left(c_{k 2}^{+}\right)^{2}\left(L_{k}^{g}\right)^{2} \frac{e^{\lambda v^{+}}}{1-\gamma}+\sum_{k=1}^{2} \xi_{k}\left(L_{k 2}^{\delta}\right)^{2} \frac{e^{\lambda \eta^{+}}}{1-\beta} \approx 1.5295 .
\end{aligned}
$$

We can check that other conditions of Theorems 1 and 2 are satisfied. So, we know that square-mean pseudo almost periodic solutions of system (46) are square-mean exponentially stable. System (46) has the initial value $u_{1}(0)=$ $0.3-0.5 i+0.6 j+0.25 k$ and $u_{2}(0)=-0.2+0.4 i-$ $0.4 j-0.35 k$. The results are verified by the numerical simulations in Figures 1-4, which shows the time response curve for the four parts between the variables $u_{1}(t)$ and $u_{2}(t)$ of system (46).

Example 2. Let $n=3$. Consider the following quaternionvalued stochastic neural network:

$$
\begin{aligned}
\mathrm{d} u_{l}(t)= & {\left[-\alpha_{l}(t) u_{l}(t)+\sum_{k=1}^{3} b_{l k}(t) f_{k}\left(u_{k}(t)\right)+\sum_{k=1}^{3} c_{l k}(t) g_{k}\left(u_{k}\left(t-v_{l k}(t)\right)\right)+\mathscr{I}_{l}(t)\right] \mathrm{d} t } \\
& +\sum_{k=1}^{3} \delta_{l k}\left(u_{k}\left(t-\eta_{l k}(t)\right)\right) \mathrm{d} W_{k}(t),
\end{aligned}
$$

where $l=1,2,3$; the coefficients are as follows: 


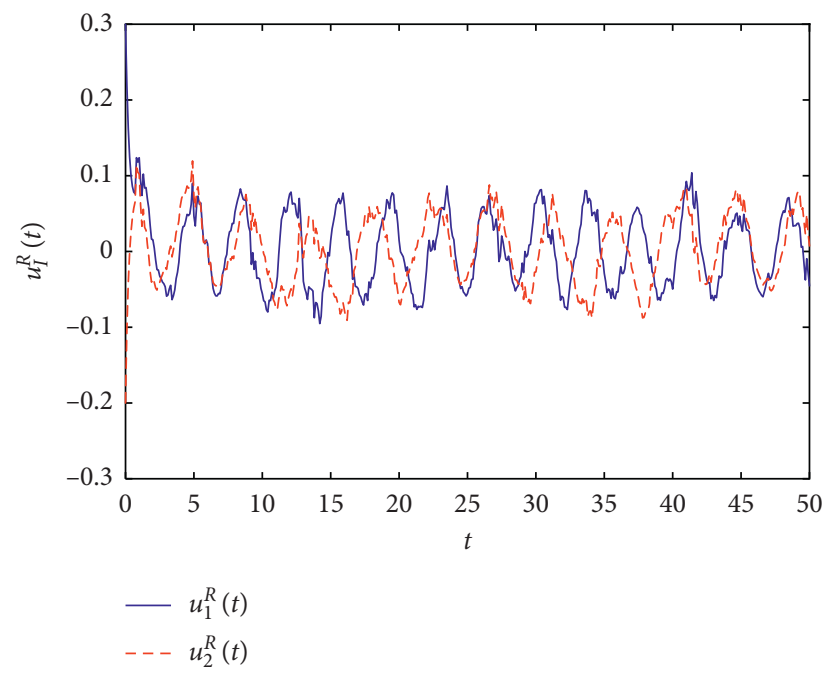

FIgURE 1: The states response of $u_{1}^{R}(t)$ and $u_{2}^{R}(t)$.

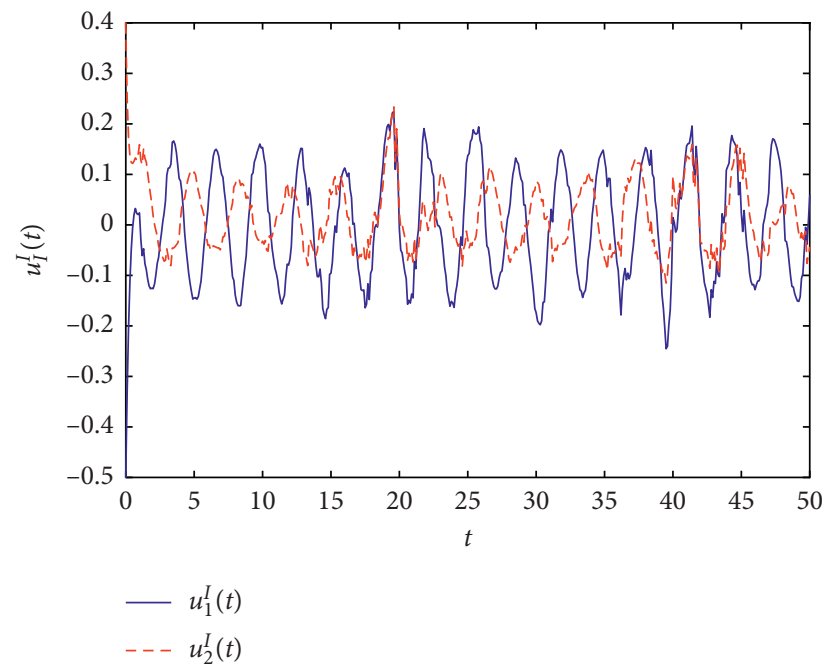

Figure 2: The states response of $u_{1}^{I}(t)$ and $u_{2}^{I}(t)$.

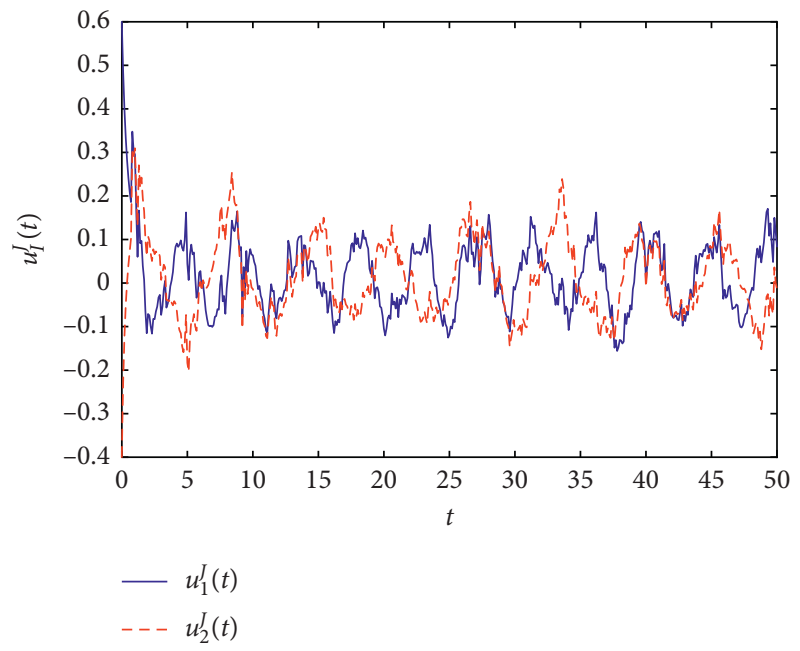

Figure 3: The states response of $u_{1}^{J}(t)$ and $u_{2}^{J}(t)$.

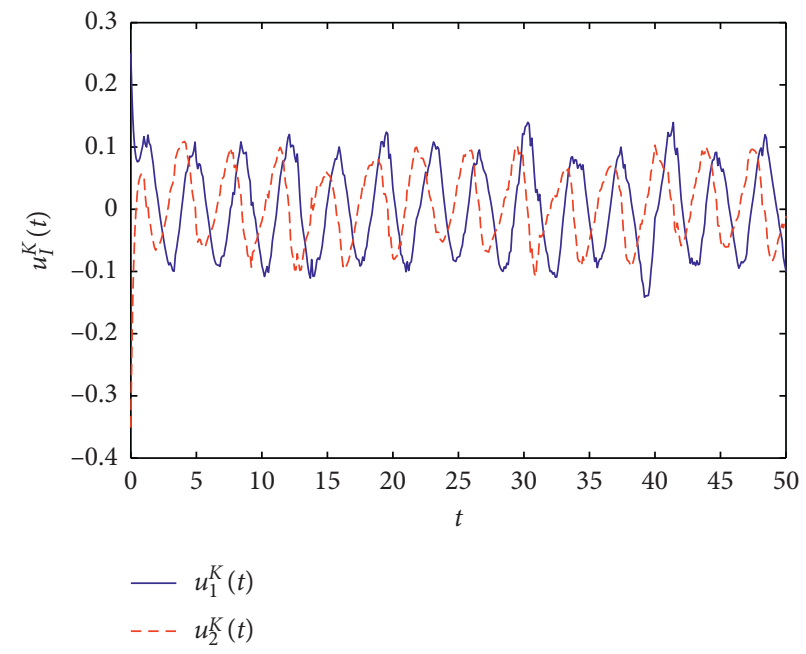

Figure 4: The states response of $u_{1}^{K}(t)$ and $u_{2}^{K}(t)$. 


$$
\begin{aligned}
f_{k}\left(u_{k}\right) & =\frac{1}{14} \sin u_{k}^{R}+i \frac{1}{12} \sin u_{k}^{I}+j \frac{1}{15} \sin \left(u_{k}^{I}+u_{k}^{J}\right)+k \frac{1}{10} \sin u_{k}^{K}, \\
g_{k}\left(u_{k}\right) & =\frac{1}{12} \sin \left(u_{k}^{R}+u_{k}^{J}\right)+i \frac{1}{20} \sin \left(u_{k}^{R}+u_{k}^{I}\right)+j \frac{1}{15} \tan h u_{k}^{J}+k \frac{1}{10} \tan h u_{k}^{K}, \\
\delta_{l k}\left(u_{k}\right) & =\frac{1}{15} \sin u_{k}^{R}+i \frac{1}{10} \sin u_{k}^{I}+j \frac{1}{8} \sin \left(u_{k}^{R}+u_{k}^{J}\right)+k \frac{1}{12} \tan h u_{k}^{K}, \\
\alpha_{1}(t) & =2+|\sin (\sqrt{3} t)| \\
\alpha_{2}(t) & =5-2 \cos (\sqrt{2} t), \\
\alpha_{3}(t) & =7-3 \cos (\sqrt{5} t), \\
b_{l k}(t) & =0.4 \cos (\sqrt{3} t)+i 0.6 \sin (\sqrt{3} t)+j 0.7 \sin t+k 0.5 \cos (2 t), \\
c_{l k}(t) & =1.2 \sin t+i 0.9 \cos (2 t)+j \sin (\sqrt{2} t)+k 1.5 \cos (\sqrt{3} t), \\
\mathscr{I}_{1}(t) & =0.2 \sin (\sqrt{3} t)+i 0.5 \cos (2 t)+j 0.3 \cos (\sqrt{2} t)+k 0.3 \sin (\sqrt{3} t), \\
\mathscr{I}_{2}(t) & \left.=0.3\left(\sin (\sqrt{2} t)+e^{-t^{2} \cos t}\right)+i 0.4 \sin (\sqrt{3} t)+j 0.5 \sin t+k 0.2 \cos (\sqrt{3} t)\right), \\
\mathscr{I}_{3}(t) & =0.2 \sin (2 t)+i 0.3 \cos (\sqrt{2} t)+j 0.4 \cos t+k 0.3 \sin (\sqrt{5} t)), \\
\eta_{k l}(t) & =\frac{1}{2}|\cos (\sqrt{2} t)|, \\
& =\frac{4}{5}|\sin (2 t)| \\
& =1,2,3 .
\end{aligned}
$$

Through simple calculations, we have 


$$
\begin{aligned}
\alpha_{1}^{-} & =2, \\
\alpha_{2}^{-} & =3, \\
\alpha_{3}^{-} & =4, \\
L_{k}^{f} & =L_{k}^{g}=\frac{1}{10}, \\
L_{l k}^{\delta} & =\frac{1}{8}, \\
v^{+} & =\frac{1}{2}, \\
\eta^{+} & =\frac{4}{5}, \\
\gamma & =\frac{1}{2}, \\
\beta & =\frac{4}{5}, \\
b_{l k}^{+} & \leq 1.1225, \\
c_{l k}^{+} & \leq 2.3452, \\
Y_{1} & \approx 0.6553, \\
Y_{3} & \approx 0.6787, \\
&
\end{aligned}
$$

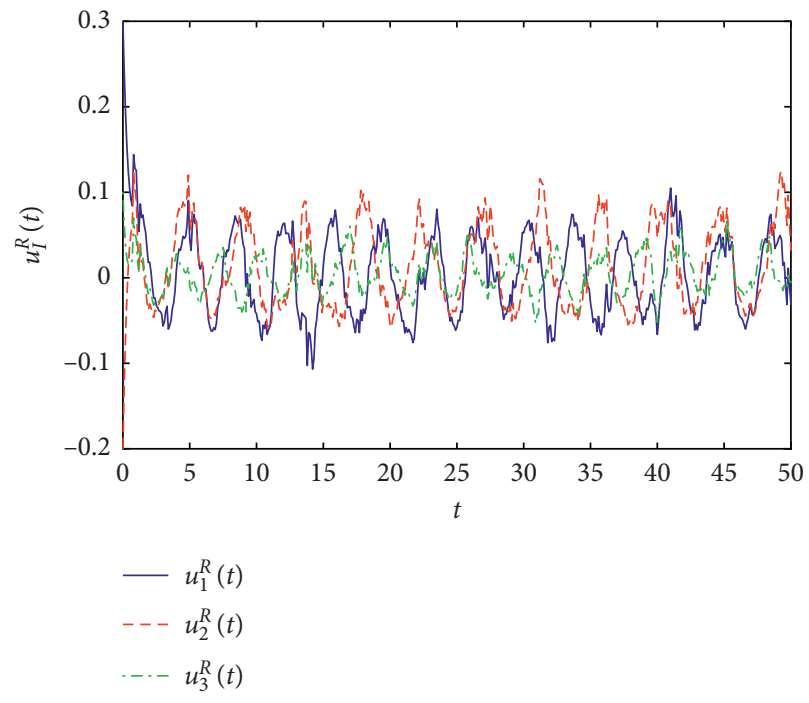

FIGURE 5: The states response of $u_{1}^{R}(t), u_{2}^{R}(t)$, and $u_{3}^{R}(t)$.

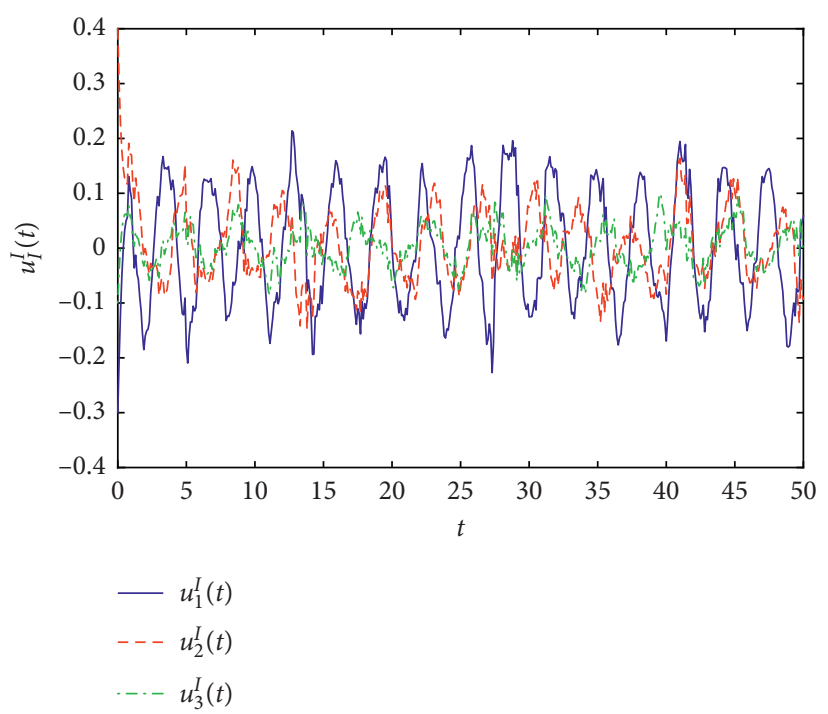

(54)

Take $\lambda=0.1, \xi_{1}=0.3, \xi_{2}=0.4$, and $\xi_{3}=0.5$, then we FIgURE 6: The states response of $u_{1}^{I}(t), u_{2}^{I}(t)$, and $u_{3}^{I}(t)$. have

Then, we have

$$
\begin{aligned}
\max \left\{\frac{3 Y_{1}}{\left(\alpha_{1}^{-}\right)^{2}}, \frac{3 Y_{2}}{\left(\alpha_{2}^{-}\right)^{2}}, \frac{3 Y_{3}}{\left(\alpha_{3}^{-}\right)^{2}}\right\} & \approx \max \{0.4915,0.2262,0.1316\} \\
& =0.4915=\rho<1 .
\end{aligned}
$$

$$
\begin{aligned}
& 2 \xi_{1} \alpha_{1}^{-}=1.2>\lambda \xi_{1}+2 \xi_{1}+\sum_{k=1}^{3} \xi_{k}\left(b_{k 1}^{+}\right)^{2}\left(L_{k}^{f}\right)^{2}+\sum_{k=1}^{3} \xi_{k}\left(c_{k 1}^{+}\right)^{2}\left(L_{k}^{g}\right)^{2} \frac{e^{\lambda v^{+}}}{1-\gamma}+\sum_{k=1}^{3} \xi_{k}\left(L_{k 1}^{\delta}\right)^{2} \frac{e^{\lambda \eta^{+}}}{1-\beta} \approx 0.8704 \\
& 2 \xi_{2} \alpha_{2}^{-}=2.4>\lambda \xi_{2}+2 \xi_{2}+\sum_{k=1}^{3} \xi_{k}\left(b_{k 2}^{+}\right)^{2}\left(L_{k}^{f}\right)^{2}+\sum_{k=1}^{3} \xi_{k}\left(c_{k 2}^{+}\right)^{2}\left(L_{k}^{g}\right)^{2} \frac{e^{\lambda v^{+}}}{1-\gamma}+\sum_{k=1}^{3} \xi_{k}\left(L_{k 2}^{\delta}\right)^{2} \frac{e^{\lambda \eta^{+}}}{1-\beta} \approx 1.0804 \\
& 2 \xi_{3} \alpha_{3}^{-}=4>\lambda \xi_{3}+2 \xi_{3}+\sum_{k=1}^{3} \xi_{k}\left(b_{k 3}^{+}\right)^{2}\left(L_{k}^{f}\right)^{2}+\sum_{k=1}^{3} \xi_{k}\left(c_{k 3}^{+}\right)^{2}\left(L_{k}^{g}\right)^{2} \frac{e^{\lambda v^{+}}}{1-\gamma}+\sum_{k=1}^{3} \xi_{k}\left(L_{k 3}^{\delta}\right)^{2} \frac{e^{\lambda \eta^{+}}}{1-\beta} \approx 1.2904 .
\end{aligned}
$$

We can check that other conditions of Theorems 1 and 2 are satisfied. So, we know that square-mean pseudo almost periodic solutions of system (51) are square-mean exponentially stable. System (51) has the initial values 


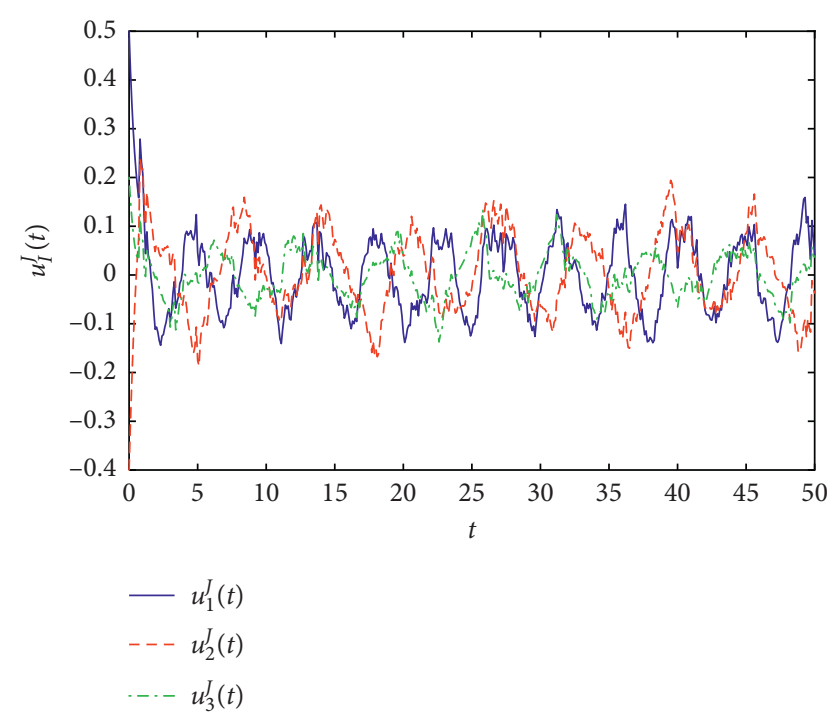

Figure 7: The states response of $u_{1}^{J}(t), u_{2}^{J}(t)$, and $u_{3}^{J}(t)$.

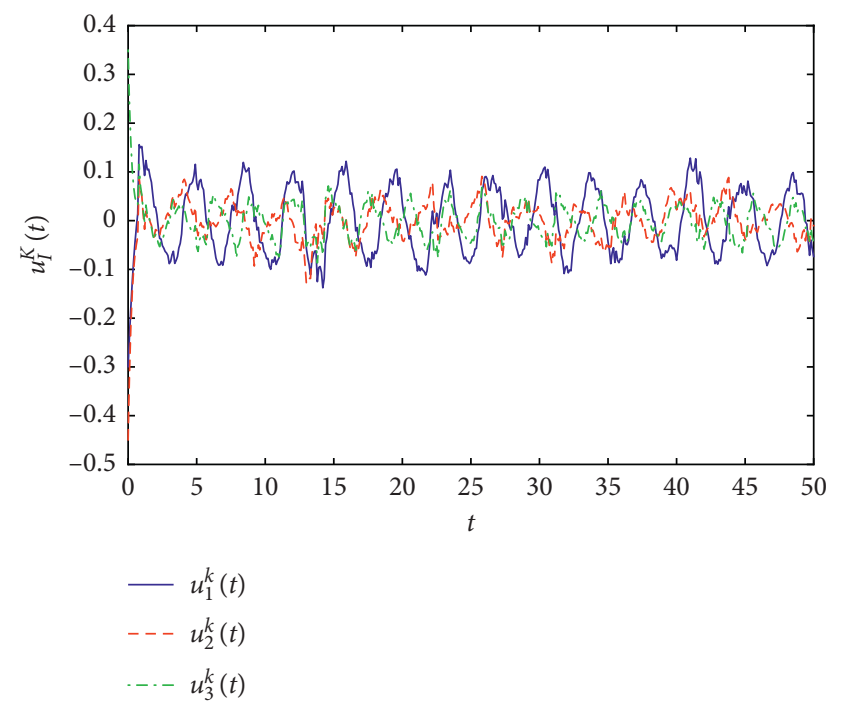

Figure 8: The states response of $u_{1}^{K}(t), u_{2}^{K}(t)$, and $u_{3}^{K}(t)$.

$u_{1}(0)=0.3-0.3 i+0.5 j-0.3 k, \quad u_{2}(0)=-0.2+0.4 i-$ $0.4 j-0.45 k$, and $u_{3}(0)=0.1-0.1 i+0.2 j+0.35 k$. The results are verified by the numerical simulations in Figures 5-8, which shows that the time response curve for the four parts between the variables $u_{1}(t), u_{2}(t)$, and $u_{3}(t)$ of system (51).

\section{Conclusion}

In this paper, we consider the existence and square-mean exponential stability of square-mean pseudo almost periodic solutions of the quaternion-valued stochastic neural networks. By using the Banach fixed point theorem and stochastic analysis techniques, we obtain some sufficient conditions for the existence of square-mean pseudo almost periodic solutions for the neural networks by direct method, and we improve the norm. Then, by constructing an appropriate Lyapunov functional, stochastic analysis theory and Itô formula, a novel sufficient condition has been derived to ensure the square-mean exponential stability for the considered stochastic neural networks. In order to demonstrate the usefulness of the presented results, some numerical examples are given. The works of this paper improve and extend the old results in literatures $[22,23]$ and propose a good research thinking to study square-mean pseudo almost periodic solutions and square-mean exponential stability of quaternion-valued stochastic neural networks with time-varying delays.

\section{Data Availability}

No data were used to support this study.

\section{Conflicts of Interest}

The authors declare that they have no conflicts of interest.

\section{Acknowledgments}

This work was supported by the Science Research Fund of Education Department of Yunnan Province of China under Grant no. 2018JS517.

\section{References}

[1] E. Wong, "Stochastic neural networks," Algorithmica, vol. 6, no. 1-6, pp. 466-478, 1991.

[2] S. Haykin, Neural Networks, Prentice-Hall, Upper Saddle River, NJ, USA, 1994.

[3] X. X. Liao and X. Mao, "Exponential stability and instability of stochastic neural networks," Stochastic Analysis and Applications, vol. 14, no. 2, pp. 165-185, 1996.

[4] S. Blythe, X. Mao, and X. Liao, "Stability of stochastic delay neural networks," Journal of the Franklin Institute, vol. 338, no. 4, pp. 481-495, 2001.

[5] H. Zhao, N. Ding, and L. Chen, "Almost sure exponential stability of stochastic fuzzy cellular neural networks with delays," Chaos, Solitons \& Fractals, vol. 40, no. 4, pp. 1653-1659, 2009.

[6] Q. Zhu and X. Li, "Exponential and almost sure exponential stability of stochastic fuzzy delayed Cohen-Grossberg neural networks," Fuzzy Sets and Systems, vol. 203, no. 2, pp. 74-94, 2012.

[7] X. Li and D. Ding, "Mean square exponential stability of stochastic Hopfield neural networks with mixed delays," Statistics \& Probability Letters, vol. 126, pp. 88-96, 2017.

[8] Z. Wang, Y. Liu, K. Fraser, and X. Liu, "Stochastic stability of uncertain Hopfield neural networks with discrete and distributed delays," Physics Letters A, vol. 354, no. 4, pp. 288-297, 2006.

[9] Z. Wang, J. A. Fang, and X. Liu, "Global stability of stochastic high-order neural networks with discrete and distributed delays," Chaos, Solitons \& Fractals, vol. 36, no. 2, pp. 388-396, 2008.

[10] R. Yang, Z. Zhang, and P. Shi, "Exponential stability on stochastic neural networks with discrete interval and distributed delays," IEEE Transactions on Neural Networks, vol. 21, no. 1, pp. 169-175, 2010.

[11] G. Chen, D. Li, L. Shi, O. van Gaans, and S. Verduyn Lunel, "Stability results for stochastic delayed recurrent neural 
networks with discrete and distributed delays," Journal of Differential Equations, vol. 264, no. 6, pp. 3864-3898, 2018.

[12] T. Isokawa, T. Kusakabe, N. Matsui, and F. Peper, Quaternion Neural Network and Its Application, Springer, Berlin, Germany, 2003.

[13] L. C. Luo, H. Feng, and L. J. Ding, "Color image compression based on quaternion neural network principal component analysis," in Proceedings of the 2010 International Conference on Multimedia Technology, ICMT 2010, Ningbo, China, October 2010.

[14] H. Shu, Q. Song, Y. Liu, Z. Zhao, and F. E. Alsaadi, "Global $\mu$-stability of quaternion-valued neural networks with nondifferentiable time-varying delays," Neurocomputing, vol. 247, pp. 202-212, 2017.

[15] Y. Liu, D. Zhang, and J. Lu, "Global exponential stability for quaternion-valued recurrent neural networks with timevarying delays," Nonlinear Dynamics, vol. 87, no. 1, pp. 553-565, 2017.

[16] X. Chen, Z. Li, Q. Song, J. Hu, and Y. Tan, "Robust stability analysis of quaternion-valued neural networks with time delays and parameter uncertainties," Neural Networks, vol. 91, pp. 55-65, 2017.

[17] Z. Tu, J. Cao, A. Alsaedi, and T. Hayat, "Global dissipativity analysis for delayed quaternion-valued neural networks," Neural Networks, vol. 89, pp. 97-104, 2017.

[18] X. F. Meng and Y. K. Li, "Pseudo almost periodic solutions for quaternion-valued cellular neural networks with discrete and distributed delays," Journal of Inequalities and Applications, vol. 2018, no. 1, p. 245, 2018

[19] C. J. Xu, M. X. Liao, P. L. Li, Z. X. Liu, and S. Yuan, "New results on pseudo almost periodic solutions of quaternionvalued fuzzy cellular neural networks with delays," Fuzzy Sets and Systems, 2020, In press.

[20] J. L. Xiang and Y. K. Li, "Pseudo almost automorphic solutions of quaternion-valued neural networks with infinitely distributed delays via a non-decomposing method," Advances in Difference Equations, vol. 2019, no. 1, p. 356, 2019.

[21] Y. K. Li, J. L. Xiang, and B. Li, "Pseudo-almost-periodic solutions of quaternion-valued RNNs with mixed delays via a direct method," Journal of Inequalities and Applications, vol. 88, pp. 1-17, 2020.

[22] R. Sriraman, G. Rajchakit, C. P. Lim, P. Chanthorn, and R. Samidurai, "Discrete-time stochastic quaternion-valued neural networks with time delays: an asymptotic stability analysis," Symmetry, vol. 12, no. 6, p. 936, 2020.

[23] U. Humphries, G. Rajchakit, P. Kaewmesri et al., "Stochastic memristive quaternion-valued neural networks with time delays: an analysis on mean square exponential input-to-state stability," Mathematics, vol. 8, no. 5, p. 815, 2020.

[24] P. H. Bezandry and T. Diagana, "Existence of almost periodic solutions to some stochastic differential equations," Applicable Analysis, vol. 86, no. 7, pp. 819-827, 2007.

[25] M. Fu and Z. Liu, "Square-mean almost automorphic solutions for some stochastic differential equations," in Proceedings of the American Mathematical Society, vol. 138, no. 10, p. 3689, 2010.

[26] Y.-K. Chang, Z.-H. Zhao, and G. M. N’Guérékata, “Squaremean almost automorphic mild solutions to non-autonomous stochastic differential equations in Hilbert spaces," Computers \& Mathematics with Applications, vol. 61, no. 2, pp. 384-391, 2011.

[27] F. Chérif, "Quadratic-mean pseudo almost periodic solutions to some stochastic differential equations in a Hilbert space,"
Journal of Applied Mathematics and Computing, vol. 40, no. 12, pp. 427-443, 2012.

[28] M. A. Diop, K. Ezzinbi, and M. M. Mbaye, "Measure theory and $S^{2}$-pseudo almost periodic and automorphic process: application to stochastic evolution equations," Afrika Matematika, vol. 26, no. 5-6, pp. 779-812, 2015.

[29] J.-X. Lu and Y. Ma, "Mean square exponential stability and periodic solutions of stochastic delay cellular neural networks," Chaos, Solitons \& Fractals, vol. 38, no. 5, pp. 1323-1331, 2008.

[30] X. Li, "Existence and global exponential stability of periodic solution for impulsive Cohen-Grossberg-type BAM neural networks with continuously distributed delays," Applied Mathematics and Computation, vol. 215, no. 1, pp. 292-307, 2009.

[31] Z. Huang and Q.-G. Yang, "Existence and exponential stability of almost periodic solution for stochastic cellular neural networks with delay," Chaos, Solitons \& Fractals, vol. 42, no. 2, pp. 773-780, 2009. 